Mannheimer Manuskripte 267

math/0210360

\title{
HIGHER GENUS AFFINE ALGEBRAS OF KRICHEVER - NOVIKOV TYPE
}

\author{
MARTIN SCHLICHENMAIER
}

\begin{abstract}
For higher genus multi-point current algebras of Krichever-Novikov type associated to a finite-dimensional Lie algebra, local Lie algebra two-cocycles are studied. They yield as central extensions almost-graded higher genus affine Lie algebras. In case that the Lie algebra is reductive a complete classification is given. For a simple Lie algebra, like in the classical situation, there is up to equivalence and rescaling only one non-trivial almost-graded central extension. The classification is extended to the algebras of meromorphic differential operators of order less or equal one on the currents algebra.
\end{abstract}

\section{INTRODUCTION}

Current algebras, their central extensions, and their representations are of great importance in mathematics, mathematical physics, and theoretical physics. These algebras supply examples of infinite dimensional Lie algebras, which are still mathematically accessible. Typically, they appear in the context of systems with infinitely many independent symmetries. For example, for Wess-Zumino-Novikov-Witten theory current algebras show up as gauge symmetry algebras. In the process of quantization of these systems one is often forced to use regularization procedures. By the "regularization" one obtains as quantum symmetry algebras central extensions of the original current algebras.

In genus zero conformal field theory, based on a finite-dimensional Lie algebra $\mathfrak{g}$, (sometimes $\mathfrak{g}$ is called horizontal algebra [12]) the current algebra is the algebra of $\mathfrak{g}$-valued Laurent polynomials $\overline{\mathfrak{g}}:=\mathfrak{g} \otimes \mathbb{C}\left[z, z^{-1}\right]$, with Lie structure

$$
\left[x \otimes z^{n}, y \otimes z^{m}\right]=[x, y] \otimes z^{n+m}, \quad x, y \in \mathfrak{g}, n, m \in \mathbb{Z} .
$$

Date: October 17, 2002.

1991 Mathematics Subject Classification. 17B67, 17B56, 17B66, 14H55, 17B65, 30F30, 81R10, $81 \mathrm{~T} 40$.

Key words and phrases. Krichever-Novikov algebras, central extensions, almost-grading, conformal field theory, infinite-dimensional Lie algebras, affine algebras, differential operator algebras, local cocycles. 
For $\mathfrak{g}$ a simple finite-dimensional Lie algebra with Cartan-Killing form (.,.) the central extension $\widehat{\mathfrak{g}}$ of the current algebra $\overline{\mathfrak{g}}$ is obtained by adding a central element $t$, i.e. $\widehat{\mathfrak{g}}=\overline{\mathfrak{g}} \oplus \mathbb{C} t$ as vector space, and replacing the above structure equation by

$$
\left[x \otimes z^{n}, y \otimes z^{m}\right]=[x, y] \otimes z^{n+m}-(x, y) \cdot n \cdot \delta_{m}^{-n} \cdot t, \quad[t, \overline{\mathfrak{g}}]=0, \quad x, y \in \mathfrak{g}, n, m \in \mathbb{Z} .
$$

This central extension $\widehat{\mathfrak{g}}$ is called an affine Lie algebra. In mathematics, e.g. they appear in the classification of Kac-Moody algebras as the untwisted algebras of affine type [17, [18. In physics they made their first appearance as symmetries of twodimensional conformal field theory models for the description of quarks, see Bardakçi and Halpern [2]. See also, e.g. [12] for further references in physics.

If $\mathfrak{g}$ is simple, it is well-known (e.g. see Garland [14]) that $\widehat{\mathfrak{g}}$ is the unique (up to equivalence and rescaling) nontrivial central extension of $\overline{\mathfrak{g}}$.

The algebras $\overline{\mathfrak{g}}$ and $\widehat{\mathfrak{g}}$ become graded Lie algebras by setting the degree to

$$
\operatorname{deg}\left(x \otimes z^{n}\right):=n, \quad \operatorname{deg} t:=0 .
$$

This grading is a crucial property in the construction of representations.

Equivalently, $\overline{\mathfrak{g}}=\mathfrak{g} \otimes \mathbb{C}\left[z, z^{-1}\right]$ can be described as the Lie algebra of $\mathfrak{g}$-valued meromorphic functions on the Riemann sphere (i.e. on the compact Riemann surface of genus 0 ) which are holomorphic outside the points 0 and $\infty$. Note that the commutative algebra $\mathbb{C}\left[z, z^{-1}\right]$ is the algebra of meromorphic functions on the sphere which are holomorphic outside 0 and $\infty$. Starting from this description of the genus zero algebra the natural extension to higher genus compact Riemann surfaces $M$ (or equivalently to smooth projective curves over $\mathbb{C}$ ) is to replace $\mathbb{C}\left[z, z^{-1}\right]$ by the commutative algebra $\mathcal{A}$ of those meromorphic functions on $M$ obeying some regularity conditions. This was done by Krichever and Novikov [22, 23, 24] for the case when the functions have poles only at two fixed points. In more details these algebras were studied by Sheinman [42, 43]. The author of the current article extended this to the case of an arbitrary but finite number of points where poles are allowed [35, 36. In this way we obtain $\overline{\mathfrak{g}}=\mathfrak{g} \otimes \mathcal{A}$, where $\mathcal{A}$ is the algebra of meromorphic functions which might have poles at the chosen set of points. The Lie algebra is given via the structure equations

$$
[x \otimes f, y \otimes g]=[x, y] \otimes f g .
$$

An essential (and non-trivial) part of these generalizations is the introduction of a graded structure. Indeed, in general it is not possible to introduce a grading, but only an almost-grading (see Definition 2.2 below). But it turns out that for the purpose of representation theory this is enough. The almost-grading is introduced by dividing the set $A$ of points where poles are allowed into two disjoint non-empty subset $I$ and $O$. Different splittings will define non-equivalent almost-gradings. Clearly, in the two-point case such a splitting and hence the almost-grading is unique.

As usual central extensions $\widehat{\mathfrak{g}}_{\gamma}$ are constructed with the help of Lie-algebra 2-cocycles $\gamma$ for the Lie algebra $\overline{\mathfrak{g}}$ with values in the trivial module $\mathbb{C}$. No other type of cocycles will appear in this article. Hence for simplicity I will call them just cocycles. 
From the point of view of representations we ask for central extensions $\widehat{\mathfrak{g}}_{\gamma}$ such that we can assign a degree to the central element in such a way that the almost-grading of $\overline{\mathfrak{g}}$ can be extended to $\widehat{\mathfrak{g}}_{\gamma}$. In contrast to the classical situation (i.e. genus zero and two marked points) not every cocycle will define a central extension which allows an extension of the almost-grading. A necessary and sufficient condition is, that the cocycle is local in the following sense. Let $\overline{\mathfrak{g}}=\mathfrak{g} \otimes \mathcal{A}=\oplus_{n \in \mathbb{Z}} \overline{\mathfrak{g}}_{n}$, be the decomposition into the homogeneous subspaces $\overline{\mathfrak{g}}_{n}$ with respect to the almost-grading. A cocycle $\gamma$ is called local, if there exist integers $L_{1}, L_{2}$, such that

$$
\gamma\left(\overline{\mathfrak{g}}_{n}, \overline{\mathfrak{g}}_{m}\right) \neq 0 \Longrightarrow L_{2} \leq n+m \leq L_{1}
$$

By assigning deg $t:=0$ (or any other integer) the almost-grading is extended to $\widehat{\mathfrak{g}}_{\gamma}$. This notion of locality of a cocycle is due to Krichever and Novikov 22].

We call a cohomology class local if it contains a representing element which is a local cocycle. Not every representing element of a local class will be local. In the classical case it turns out, that every cocycle class is local. This is neither the case if we allow more points nor if we consider Riemann surfaces of higher genus. The main goal of this article is to give a complete classification of local cocycles for the higher genus and multipoint current algebras at least in the case when the finite-dimensional Lie algebra $\mathfrak{g}$ is reductive. In this way the almost-graded (higher genus and multi-point) affine algebras are classified as central extensions of current algebras up to equivalence. Sometimes central extensions defined via local cocycles are called local central extensions. To avoid misinterpretations I prefer to use almost-graded central extensions instead. It should be pointed out that the cocycles obtained by projective representations are local in all important cases, e.g. in the case of semi-infinite wedge representations.

The results obtained in this article are described as follows. Let $\mathfrak{g}=\mathfrak{g}_{0} \oplus \mathfrak{g}_{1} \oplus \cdots \oplus \mathfrak{g}_{M}$ be a finite-dimensional reductive Lie algebra with abelian summand $\mathfrak{g}_{0}$ of dimension $n$ and $M$ simple summands $\mathfrak{g}_{1}, \ldots, \mathfrak{g}_{M}$. Let $C_{S}$ be a cycle separating the points in $I$ from the points in $O$ (see near Equation (2.4) for more information on its definition). First we consider the semisimple case. It is shown that for every local cocycle $\gamma$ of $\overline{\mathfrak{g}}$ we can find a symmetric invariant bilinear form $\alpha$ on $\mathfrak{g}$ such that $\gamma$ is cohomologous to

$$
\gamma^{\prime}(x \otimes f, y \otimes g)=\frac{\alpha(x, y)}{2 \pi \mathrm{i}} \int_{C_{S}} f d g .
$$

Vice versa, every such cocycle is local. The corresponding central extension is equivalent to the central extension defined by

$$
[x \otimes f, y \otimes g]:=[x, y] \otimes(f g)+\frac{\alpha(x, y)}{2 \pi \mathrm{i}} \int_{C_{S}} f d g .
$$

This will imply that the space $\mathrm{H}_{\text {loc }}^{2}(\overline{\mathfrak{g}}, \mathbb{C})$ of local cocycle classes is $M$-dimensional, see Theorem 3.13 and Theorem 3.16. In particular, for the current algebra associated to a simple Lie algebra we obtain that there is up to equivalence and rescaling only one 
non-trivial almost-graded central extension. In this case $\alpha$ is necessarily the CartanKilling form, and is non-degenerate. Let me again stress the fact, that without the locality assumption for the cocycle (corresponding to the almost-gradedness of the central extension) the statement would be wrong. The only exception is the classical affine Kac-Moody case.

An important concept shows up, the notion of $\mathcal{L}$-invariance of a cocycle. Here $\mathcal{L}$ denotes the Lie algebra of meromorphic vector fields on $M$, holomorphic outside the points in $A$. A cocycle is called $\mathcal{L}$-invariant if

$$
\gamma(x \otimes(e . f), y \otimes g)+\gamma(x \otimes f, y \otimes(e . g))=0, \quad, \forall x, y \in \mathfrak{g}, f, g \in \mathcal{A}, e \in \mathcal{L} .
$$

Here $e . g$ denotes the (Lie-)derivative of $g$ with respect to the vector field $e$. I will show (see Theorem 3.16) that in the semisimple case in any local cohomology class there is a unique $\mathcal{L}$-invariant representing local cocycle. It will be given by (1.6). This result might be compared with the notion of Diff $S^{1}$-invariant cocycles in the $C^{\infty}$-context studied by Pressley and Segal [28].

In the semisimple case, restricting the attention to $\mathcal{L}$-invariant cocycles does not reduce the space of cocycle classes. This is different in the general reductive case. Already in the case of pure abelian $\mathfrak{g}$, e.g. for $\overline{\mathfrak{g}}=\mathcal{A}$, we obtain many linearly independent local cocycles. Because $\overline{\mathfrak{g}}$ is abelian they cannot be cohomologous. But if we assume for the cocycle under consideration that its restriction to $\overline{\mathfrak{g}}_{0} \times \overline{\mathfrak{g}}_{0}$ is $\mathcal{L}$-invariant, we obtain the same form of the classification result as in the semisimple case. Again a symmetric invariant bilinear form $\alpha$ classifies those local cohomology classes, and the central extensions are equivalent to central extensions given by (1.7). The condition of invariance for $\alpha$ is restricted to $\mathfrak{g}_{0}$ void. Hence, the cohomology space $\mathrm{H}_{l o c, \mathcal{L}}^{2}(\overline{\mathfrak{g}}, \mathbb{C})$ has dimension

$$
\frac{n(n+1)}{2}+M, \quad n=\operatorname{dim} \mathfrak{g}_{0}, \quad M \text { is the number of simple summands. }
$$

For non-reductive $\mathfrak{g}$ certain results are obtained. But there is no complete classification.

In the context of fermionic or semi-infinite wedge representations of $\overline{\mathfrak{g}}$, resp. $\widehat{\mathfrak{g}}$, there is always a representation of a central extension of the differential operator algebra $\mathcal{D}_{\mathfrak{g}}^{1}$ of operators of degree $\leq 1$ around. The algebra $\mathcal{D}_{\mathfrak{g}}^{1}$ is a semi-direct sum of $\overline{\mathfrak{g}}$ and $\mathcal{L}$ induced by the action of $\mathcal{L}$ on $\overline{\mathfrak{g}}$, i.e. $\mathcal{D}_{\mathfrak{g}}^{1}=\overline{\mathfrak{g}} \oplus \mathcal{L}$ as vector space with given Lie structure on the summands, and $[e, x \otimes f]=x \otimes(e . f)$ for $e \in \mathcal{L}, x \in \mathfrak{g}, f \in \mathcal{A}$. For $\mathfrak{g}=\mathbb{C}$ this algebra is the standard algebra of meromorphic differential operators of degree $\leq 1$ with poles at most at the points in $A$. For this algebra the local cocycle classes have been determined in [38]. The space of local cocycle classes is 3-dimensional. It is generated by a standard cocycle $\gamma_{S}^{(f)}$ for the function algebra $\mathcal{A}$, a standard cocycle $\gamma_{S}^{(v)}$ for the vector field algebra $\mathcal{L}$, and a mixing cocycle $\gamma_{S}^{(m)}$. The results are recalled in Section 2 from 38. In the classical case the corresponding result was shown by Arbarello, De Concini, Kac, and Procesi [1.

Using these results and the classification result for the cocycles of $\overline{\mathfrak{g}}$ for $\mathfrak{g}$ reductive, with some additional work, a complete classification of local cocycles of $\mathcal{D}_{\mathfrak{g}}^{1}$ is given. 
It turns out, that every local cocycle $\gamma$ is cohomologous to a linear combination of a local (and $\mathcal{L}$-invariant) cocycle for $\overline{\mathfrak{g}}$, of the vector field cocycle $\gamma_{S}^{(v)}$, and a cocycle $\gamma_{\phi}$ of mixing type, defined by

$$
\gamma_{\phi}(e, x \otimes g)=\phi(x) \cdot \gamma_{S}^{(m)}(e, g), \quad e \in \mathcal{L}, g \in A, x \in \mathfrak{g} .
$$

Here $\phi$ is a linear form on $\mathfrak{g}$ vanishing on the derived subalgebra $\mathfrak{g}^{\prime}=[\mathfrak{g}, \mathfrak{g}]$, i.e. on the semisimple complement of $\mathfrak{g}_{0}$. It is understood that all these basic cocycles are extended by zero on the corresponding complementary subspaces. It should be noted that the condition of $\mathcal{L}$-invariance of $\gamma$ restricted to $\overline{\mathfrak{g}}_{0}$ is automatically fulfilled, as $\gamma$ is a cocycle for $\mathcal{D}_{\mathfrak{g}}^{1}$, see Proposition 4.10. Note also, that if $\mathfrak{g}$ is a semisimple Lie algebra the linear form $\phi$ has to vanish identically, hence there will be no cohomologically nontrivial cocycles of mixing type. The absence of the mixing cocycle in the semisimple case was also shown by Sheinman in 45 .

Such central extensions $\widehat{\mathcal{D}_{\mathfrak{g}}^{1}}$ of $\mathcal{D}_{\mathfrak{g}}^{1}$ show also up in the context of the Sugawara representations associated to representations of higher genus affine algebras [40, 37.

Algebras of the type $\overline{\mathfrak{g}}=\mathfrak{g} \otimes \mathcal{A}$ for an arbitrary commutative algebra $A$ and $\mathfrak{g}$ a finite-dimensional simple Lie algebra, and their central extensions were considered earlier from the purely algebraic point of view by Kassel [19, and Kassel and Loday 20]. In particular, no grading was considered. Hence the question, which cocycles are local and define (almost)-graded central extensions could also not be considered. In [19] for $\mathfrak{g}$ simple with the help of the Kähler differentials of $\mathcal{A}$ the universal central extension of $\overline{\mathfrak{g}}$ was constructed. Indeed, I use this universal central extension in the proof of Theorem 3.13. It should be remarked that Bremner 6, 7] gave an explicit description of the universal central extensions in the genus zero case with four marked points, and in the genus one case with two marked points. For completeness let me note that by Santharoubane [31, Haddi [16], Zusmanovich 48, Berman and Kryliouk [4] the result of Kassel, suitably modified, has been extended to semisimple $\mathfrak{g}$ and perfect $\mathfrak{g}$ respectively. But please note the fact that none of these more general results on universal central extensions will be used here to classify the local cocycles.

As a remark aside, not directly related to the topic of this article, let me add that universal central extensions in the case that $\mathcal{A}$ is the algebra of $C^{\infty}$-functions on a manifold are studied for example by Pressley and Segal [28, Etingof and Frenkel [8], Frenkel and Khesin [11, and Roger [29]. If $\mathcal{A}$ is a toroidal Lie algebra, i.e. an algebra of Laurent polynomials in $N$ variables, the universal central extensions and representations of it have been studied in more detail. For example see Moody, Rao and Yokonoma [27, Berman and Billig [3], and Larsson [25].

Determining the cohomology of infinite-dimensional Lie algebras is an important and demanding task. In the context of classical current algebras let me mention the work of Feigin [9] beside the references already quoted above. There is a huge literature on the cohomology of vector field algebras, e.g. see the book of Fuks [13] which contains a long list of further references. For the meromorphic vector field algebras related 
to higher genus Riemann surfaces (i.e. the Krichever-Novikov vector field algebras) the importance of local cocycles was realized by Krichever and Novikov [22, 23, 24]. They showed in the case of two marked points for these algebras the uniqueness of the local cocycle class. See [38] for generalization of the result. For work in direction of determining the full cohomology of the Krichever-Novikov vector field algebras see for example the work of Feigin [10], Kawazumi [21], and Wagemann [46, 47].

The structure of this article is as follows. In Section 2 the basic definitions of the multi-point higher genus function, vector field, and differential operator algebras of Krichever-Novikov type are recalled. The classification results (uniqueness, etc.) of local cocycles for these algebras are quoted from 38]. In Section 3 the definition of the higher genus affine algebras for general Lie algebras are given. The above mentioned classification results for the reductive case are shown. In Section 4 the algebras $\mathcal{D}_{\mathfrak{g}}^{1}$ of differential operators of degree $\leq 1$ for the current algebra $\overline{\mathfrak{g}}$ are studied. Finally, in Section 5 the algebras associated to $\mathfrak{g l}(n)$ and $\mathfrak{s l}(n)$ are considered as examples .

It is a pleasure for me to thank Oleg Sheinman whose questions inspired me to attack systematically the problem of almost-graded central extensions of current algebras, not only for the current algebras of simple Lie algebras. He also pointed out to me the importance of the algebras $\widehat{\mathcal{D}_{\mathfrak{g}}^{1}}$ in the context of fermionic representations [44, 45]. Discussions on universal central extensions with Karl-Hermann Neeb and Friedrich Wagemann at the Workshop on Lie Theory 2002 in Darmstadt are acknowledged. Even if the topic of the discussion finally did not show up in this work directly, it helped to clear up my thoughts.

\section{The multi-Point algebras of Krichever-Novikov type}

In this section I recall the basic set-up and definitions for the associative algebra of functions, the Lie algebra of vector fields, and the Lie algebra of differential operators of Krichever - Novikov type, including their central extensions. In particular, the uniqueness results obtained in [38] and needed further down are recalled. The introduction of the current algebra, of the affine algebra, and of the Lie algebra valued differential operator algebra associated to the current algebra will be postponed to the next sections.

2.1. Geometric set-up and the involved algebras. Let $M$ be a compact Riemann surface of genus $g$, or in terms of algebraic geometry, a smooth projective curve over C. Let $N, K \in \mathbb{N}$ with $N \geq 2$ and $1 \leq K<N$. Fix $I=\left(P_{1}, \ldots, P_{K}\right)$, and $O=\left(Q_{1}, \ldots, Q_{N-K}\right)$ disjoint ordered tuples of distinct points ("marked points", "punctures") on the Riemann surface. In particular, we assume $P_{i} \neq Q_{j}$ for every pair $(i, j)$. The points in $I$ are called the in-points, the points in $O$ the out-points. Sometimes I will consider $I$ and $O$ simply as sets and use $A=I \cup O$.

Let $\mathcal{K}$ be the canonical line bundle of $M$. Its associated sheaf of local sections is the sheaf of holomorphic differentials. Following the common practice, I will usually not distinguish between a line bundle and its associated invertible sheaf of section. For 
every $\lambda \in \mathbb{Z}$ we consider the bundle $\mathcal{K}^{\lambda}:=\mathcal{K}^{\otimes \lambda}$. Here I use the usual convention: $\mathcal{K}^{0}:=\mathcal{O}$ is the trivial bundle, and $\mathcal{K}^{-1}:=\mathcal{K}^{*}$ is the holomorphic tangent line bundle (resp. the sheaf of holomorphic vector fields). After fixing a theta characteristics, i.e. a bundle $S$ with $S^{\otimes 2}=\mathcal{K}$, we can also consider $\lambda \in \frac{1}{2} \mathbb{Z}$ with respect to the chosen theta characteristics. In this article we will only need $\lambda \in \mathbb{Z}$. Denote by $\mathcal{F}^{\lambda}$ the (infinitedimensional) vector space ${ }^{1}$ of global meromorphic sections of $\mathcal{K}^{\lambda}$ which are holomorphic on $M \backslash A$.

Special cases, which are of particular interest to us, are the quadratic differentials $(\lambda=2)$, the differentials $(\lambda=1)$, the functions $(\lambda=0)$, and the vector fields $(\lambda=-1)$. The space of functions will be denoted by $\mathcal{A}$ and the space of vector fields by $\mathcal{L}$. By multiplying sections with functions we again obtain sections. In this way the space $\mathcal{A}$ becomes an associative algebra and the spaces $\mathcal{F}^{\lambda}$ become $\mathcal{A}$-modules.

The vector fields in $\mathcal{L}$ operate on $\mathcal{F}^{\lambda}$ by taking the Lie derivative $L$. In local coordinates

$$
L_{e}(g)_{\mid}:=\left(\tilde{e}(z) \frac{d}{d z}\right) \cdot\left(\tilde{g}(z) d z^{\lambda}\right):=\left(\tilde{e}(z) \frac{d \tilde{g}}{d z}(z)+\lambda \tilde{g}(z) \frac{d \tilde{e}}{d z}(z)\right) d z^{\lambda} .
$$

Here $e \in \mathcal{L}$ and $g \in \mathcal{F}^{\lambda}$, and $\tilde{e}$ and $\tilde{g}$ are their local representing functions.

The space $\mathcal{L}$ becomes a Lie algebra with respect to the Lie derivative (2.1) and the spaces $\mathcal{F}^{\lambda}$ become Lie modules over $\mathcal{L}$. As usual I write $[e, f]$ for the bracket of the vector fields.

For the Riemann sphere $(g=0)$ with quasi-global coordinate $z$ and $I=(0)$ and $O=$ $(\infty)$, the introduced function algebra is the algebra of Laurent polynomials $\mathbb{C}\left[z, z^{-1}\right]$, and the vector field algebra is the Witt algebra, i.e. the algebra whose universal central extension is the Virasoro algebra. We denote for short this situation as the classical situation.

The vector field algebra $\mathcal{L}$ operates on the algebra $\mathcal{A}$ of functions as derivations. Hence it is possible to consider the semi-direct sum $\mathcal{D}^{1}=\mathcal{A} \rtimes \mathcal{L}$. This Lie algebra is the algebra of meromorphic differential operators of degree $\leq 1$ which are holomorphic on $M \backslash A$. As vector space $\mathcal{D}^{1}=\mathcal{A} \oplus \mathcal{L}$ and the Lie product is given as

$$
[(g, e),(h, f)]:=(e \cdot h-f \cdot g,[e, f]) .
$$

There is the short exact sequence of Lie algebras

$$
0 \longrightarrow \mathcal{A} \stackrel{i_{1}}{\longrightarrow} \mathcal{D}^{1} \stackrel{p_{2}}{\longrightarrow} \mathcal{L} \longrightarrow 0 .
$$

Obviously, $\mathcal{L}$ is also a subalgebra of $\mathcal{D}^{1}$. The vector spaces $\mathcal{F}^{\lambda}$ become $\mathcal{D}^{1}$-modules by the canonical definition $(g, e) \cdot v=g \cdot v+e \cdot v, \quad v \in \mathcal{F}^{\lambda}$. By universal constructions algebras of differential operators of arbitrary degree can be considered [33, 35, [39]. There is another type of algebra of importance, the current algebra. It will be defined in Section 3 .

\footnotetext{
${ }^{1}$ For $\lambda=\frac{1}{2}+\mathbb{Z}$ we should denote the vector space by $\mathcal{F}_{S}^{\lambda}$ and let $S$ go through all theta characteristics.
} 
Let $\rho$ be a meromorphic differential which is holomorphic on $M \backslash A$ with exact pole order 1 at the points in $A$, given positive residues at $I$, given negative residues at $O$ (of course obeying the restriction $\left.\sum_{P \in I} \operatorname{res}_{P}(\rho)+\sum_{Q \in O} \operatorname{res}_{Q}(\rho)=0\right)$, and purely imaginary periods. There exists exactly one such $\rho$ (see [32, p.116]). For $R \in M \backslash A$ a fixed point, the function $u(P)=\operatorname{Re} \int_{R}^{P} \rho$ is a well-defined harmonic function. The family of level lines $C_{\tau}:=\{P \in M \mid u(P)=\tau\}, \tau \in \mathbb{R}$, defines a fibration of $M \backslash A$. Each $C_{\tau}$ separates the points in $I$ from the points in $O$. For $\tau \ll 0(\tau \gg 0)$ each level line $C_{\tau}$ is a disjoint union of deformed circles $C_{i}$ around the points $P_{i}, i=1, \ldots, K$ (of deformed circles $C_{i}^{*}$ around the points $\left.Q_{i}, i=1, \ldots, N-K\right)$.

For $f \in \mathcal{F}^{\lambda}$ and $g \in \mathcal{F}^{\mu}$ we have $f \otimes g \in \mathcal{F}^{\lambda+\mu}$. In particular, for $\mu=1-\lambda$ the form $f \otimes g$ is a meromorphic differential.

Definition 2.1. The Krichever-Novikov pairing (KN pairing) is the pairing between $\mathcal{F}^{\lambda}$ and $\mathcal{F}^{1-\lambda}$ given by

$$
\begin{gathered}
\mathcal{F}^{\lambda} \times \mathcal{F}^{1-\lambda} \rightarrow \mathbb{C}, \\
\langle f, g\rangle:=\frac{1}{2 \pi \mathrm{i}} \int_{C_{\tau}} f \otimes g=\sum_{P \in I} \operatorname{res}_{P}(f \otimes g)=-\sum_{Q \in O} \operatorname{res}_{Q}(f \otimes g),
\end{gathered}
$$

where $C_{\tau}$ is any non-singular level line.

The last equalities follow from the residue theorem. Note that in (2.4) the integral does not depend on the level line chosen. We will call any such level line or any cycle cohomologous to such a level line a separating cycle $C_{S}$.

2.2. Almost-graded structure. For infinite dimensional algebras and their representation theory a graded structure is usually of importance to obtain structure results. A typical example is given by the Witt algebra $W . W$ admits a preferred set of basis elements given by $\left\{e_{n}=z^{n+1} \frac{d}{d z} \mid n \in \mathbb{Z}\right\}$. One calculates $\left[e_{n}, e_{m}\right]=(m-n) e_{n+m}$. Hence $\operatorname{deg}\left(e_{n}\right):=n$ makes $W$ to a graded Lie algebra.

In our more general context the algebras will almost never be graded. But it was observed by Krichever and Novikov in the two-point case that a weaker concept, an almost-graded structure (they call it a quasi-graded structure), will be enough to develop an interesting theory of representations (highest weight representations, Verma modules, etc.).

Definition 2.2. (a) Let $\mathcal{L}$ be an (associative or Lie) algebra admitting a direct decomposition as vector space $\mathcal{L}=\bigoplus_{n \in \mathbb{Z}} \mathcal{L}_{n}$. The algebra $\mathcal{L}$ is called an almost-graded algebra if (1) $\operatorname{dim} \mathcal{L}_{n}<\infty$ and (2) there are constants $R$ and $S$ with

$$
\mathcal{L}_{n} \cdot \mathcal{L}_{m} \subseteq \bigoplus_{h=n+m+R}^{n+m+S} \mathcal{L}_{h}, \quad \forall n, m \in \mathbb{Z} .
$$

The elements of $\mathcal{L}_{n}$ are called homogeneous elements of degree $n$.

(b) Let $\mathcal{L}$ be an almost-graded (associative or Lie) algebra and $\mathcal{M}$ an $\mathcal{L}$-module with 
decomposition $\mathcal{M}=\bigoplus_{n \in \mathbb{Z}} \mathcal{M}_{n}$ as vector space. The module $\mathcal{M}$ is called an almostgraded module, if (1) $\operatorname{dim} \mathcal{M}_{n}<\infty$, and (2) there are constants $R^{\prime}$ and $S^{\prime}$ with

$$
\mathcal{L}_{m} \cdot \mathcal{M}_{n} \subseteq \bigoplus_{h=n+m+R^{\prime}}^{n+m+S^{\prime}} \mathcal{M}_{h}, \quad \forall n, m \in \mathbb{Z}
$$

The elements of $\mathcal{M}_{n}$ are called homogeneous elements of degree $n$.

For the 2-point situation for $M$ a higher genus Riemann surface and $I=\{P\}$, $O=\{Q\}$ with $P, Q \in M$, Krichever and Novikov [22] introduced an almost-graded structure of the algebras and the modules by exhibiting special bases and defining their elements to be the homogeneous elements. In [33, 34 its multi-point generalization was given, again by exhibiting a special basis. (See also Sadov 30, for some results in similar directions.)

In more detail, for fixed $\lambda$ and for every $n \in \mathbb{Z}$, and $i=1, \ldots, K$, a certain element $f_{n, p}^{\lambda} \in \mathcal{F}^{\lambda}$ is exhibited in [33, 34]. The $f_{n, p}^{\lambda}$ for $p=1, \ldots, K$ are a basis of a subspace $\mathcal{F}_{n}^{\lambda}$ and it is shown that

$$
\mathcal{F}^{\lambda}=\bigoplus_{n \in \mathbb{Z}} \mathcal{F}_{n}^{\lambda}
$$

The subspace $\mathcal{F}_{n}^{\lambda}$ is called the homogeneous subspace of degree $n$.

The basis elements are chosen in such a way that they fulfill the duality relation with respect to the $\mathrm{KN}$ pairing (2.4)

$$
\left\langle f_{n, p}^{\lambda}, f_{m, r}^{1-\lambda}\right\rangle=\delta_{-n}^{m} \cdot \delta_{p}^{r} .
$$

This implies that the KN pairing is non-degenerate.

We will need as additional information about the elements $f_{n, p}^{\lambda}$ that

$$
\operatorname{ord}_{P_{i}}\left(f_{n, p}^{\lambda}\right)=(n+1-\lambda)-\delta_{i}^{p}, \quad i=1, \ldots, K .
$$

The recipe for choosing the orders at the points in $O$ is such that up to a scalar multiplication there is a unique such element which also fulfills (2.7). After choosing local coordinates $z_{p}$ at the points $P_{p}$ the scalar can be fixed by requiring

$$
f_{n, p \mid}^{\lambda}\left(z_{p}\right)=z_{p}^{n-\lambda}\left(1+O\left(z_{p}\right)\right)\left(d z_{p}\right)^{\lambda} .
$$

We introduce the following notation:

$$
A_{n, p}:=f_{n, p}^{0}, \quad e_{n, p}:=f_{n, p}^{-1}, \quad \omega^{n, p}:=f_{-n, p}^{1}, \quad \Omega^{n, p}:=f_{-n, p}^{2} .
$$

A detailed analysis [33, 34] using (2.7) yields

Theorem 2.3. With respect to the above introduced grading the associative algebra $\mathcal{A}$, and the Lie algebras $\mathcal{L}$ and $\mathcal{D}^{1}$ are almost-graded algebras and the modules $\mathcal{F}^{\lambda}$ are almost-graded modules over them. In all cases the lower shifts in the degree of the result (i.e. the numbers $R, R^{\prime}$ in (2.5) and (2.6)) are zero. 
Let us abbreviate the terms of higher degrees as the one under consideration with the symbol h.d.t.. By calculating the exact residues in the case of the lower bound we obtain

Proposition 2.4.

$$
\begin{array}{ll}
A_{n, p} \cdot A_{m, r}=\delta_{p}^{r} \cdot A_{n+m, r}+\text { h.d.t., } & A_{n, p} \cdot f_{m, r}^{\lambda}=\delta_{p}^{r} \cdot f_{n+m, r}^{\lambda}+\text { h.d.t., } \\
{\left[e_{n, p}, e_{m, r}\right]=\delta_{p}^{r} \cdot(m-n) \cdot e_{n+m, r}+\text { h.d.t., }} & e_{n, p} \cdot f_{m, r}^{\lambda}=\delta_{p}^{r} \cdot(m+\lambda n) \cdot f_{n+m, r}^{\lambda}+\text { h.d.t.. }
\end{array}
$$

2.3. Central extensions and local cocycles. Let $\mathcal{V}$ be a Lie algebra and $\gamma$ a Lie algebra two-cocycle of $\mathcal{V}$ with values in $\mathbb{C}$, i.e. $\gamma$ is an antisymmetric bilinear form on $\mathcal{V}$ obeying

$$
\gamma([f, g], h)+\gamma([g, h], f)+\gamma([h, f], g)=0, \quad \forall f, g, h \in \mathcal{V} .
$$

On $\widehat{\mathcal{V}}:=\widehat{\mathcal{V}}_{\gamma}=\mathbb{C} \oplus \mathcal{V}$ a Lie algebra structure can be defined by (with the notation $\widehat{f}:=(0, f)$ and $t:=(1,0))$

$$
[\widehat{f}, \widehat{g}]:=\widehat{[f, g]}+\gamma(f, g) \cdot t, \quad[t, \widehat{\mathcal{V}}]=0 .
$$

The element $t$ is a central element. The algebra $\widehat{\mathcal{V}}_{\gamma}$ is called a central extension of $\mathcal{V}$. Up to equivalence central extensions are classified by the elements of $\mathrm{H}^{2}(\mathcal{V}, \mathbb{C})$, the second Lie algebra cohomology space with values in the trivial module $\mathbb{C}$. In particular, two cocycles $\gamma_{1}, \gamma_{2}$ define equivalent central extensions if and only if there exist a linear form $\phi$ on $\mathcal{V}$ such that

$$
\gamma_{1}(f, g)=\gamma_{2}(f, g)+\phi([f, g])
$$

Given a linear form $\phi$ on $\mathcal{V}$ we denote the cocycle obtained as coboundary by $\phi$ by the symbol $\delta \Phi$, i.e.

$$
\delta \phi(f, g):=\phi([f, g]) .
$$

Definition 2.5. Let $\mathcal{V}=\bigoplus_{n \in \mathbb{Z}} \mathcal{V}_{n}$ be an almost-graded Lie algebra. A cocycle $\gamma$ for $\mathcal{V}$ is called local (with respect to the almost-grading) if there exist $M_{1}, M_{2} \in \mathbb{Z}$ with

$$
\forall n, m \in \mathbb{Z}: \quad \gamma\left(\mathcal{V}_{n}, \mathcal{V}_{m}\right) \neq 0 \Longrightarrow M_{2} \leq n+m \leq M_{1}
$$

By defining $\operatorname{deg}(t):=0$ (or any other integer), the central extension $\widehat{\mathcal{V}}_{\gamma}$ is almostgraded if and only if it is given by a local cocycle $\gamma$. In this case we call $\widehat{\mathcal{V}}_{\gamma}$ an almost-graded central extension (sometimes also called local central extension).

In the following we consider cocycles of geometric origin for the algebras introduced above. A thorough treatment for them is given in 38]. The proofs of the following statements and more details can be found there.

For the abelian Lie algebra $\mathcal{A}$ any antisymmetric bilinear form will be a 2-cocycle. Let $C$ be any (not necessarily connected) differentiable cycle in $M \backslash A$ then

$$
\gamma_{C}^{(f)}: \mathcal{A} \times \mathcal{A} \rightarrow \mathbb{C}, \quad \gamma_{C}^{(f)}(g, h):=\frac{1}{2 \pi \mathrm{i}} \int_{C} g d h
$$


is antisymmetric, hence a cocycle. Note that by replacing $C$ by any homologous (differentiable) cycle one obtains the same cocycle. The above cocycle is $\mathcal{L}$-invariant, i.e.

$$
\gamma_{C}^{(f)}(e \cdot g, h)+\gamma_{C}^{(f)}(g, e \cdot h)=0, \quad \forall e \in \mathcal{L}, \forall g, h \in \mathcal{A},
$$

and it is multiplicative, i.e.

$$
\gamma(f g, h)+\gamma(g h, f)+\gamma(h f, g)=0, \quad \forall f, g, h \in \mathcal{A} .
$$

To generalize the Virasoro-Gelfand-Fuks cocycle $\oint\left(e^{\prime \prime \prime} f-e f^{\prime \prime \prime}\right)$ for the Witt algebra to higher genus vector field algebras $\mathcal{L}$ one has first to choose a projective connection and add a counter term to the integrand to obtain a well-defined 1-differential which can be integrated. Let $R$ be a global holomorphic projective connection, see e.g. 38, for the definition. For every cycle $C$ (and every $R$ ) a cocycle is given by

$$
\gamma_{C, R}^{(v)}(e, f):=\frac{1}{24 \pi \mathrm{i}} \int_{C}\left(\frac{1}{2}\left(\tilde{e}^{\prime \prime \prime} \tilde{f}-\tilde{e} \tilde{f}^{\prime \prime \prime}\right)-R \cdot\left(\tilde{e}^{\prime} \tilde{f}-\tilde{e} \tilde{f}^{\prime}\right)\right) d z
$$

Here $e_{\mid}=\tilde{e} \frac{d}{d z}$ and $f_{\mid}=\tilde{f} \frac{d}{d z}$ with local meromorphic functions $\tilde{e}$ and $\tilde{f}$. A different choice of the projective connection (even if we allow meromorphic projective connections with poles possibly at the points in $A$ ) yields a cohomologous cocycle, hence an equivalent central extension.

These two types of cocycles can be extended to cocycles of $\mathcal{D}^{1}$ by setting them to be zero if one of the entries are from the complementary space. For the vector field cocycles this is clear, because it is obtained by pulling back the cocycle to $\mathcal{D}^{1}$ via (2.3). For the extension of the function algebra cocycles the $\mathcal{L}$-invariance (2.17) is crucial. But there are other independent types of cocycles which mix functions with vector fields. To define them we first have to fix an affine connection $T$ which is holomorphic outside $A$ and has at most a pole of order one at the point $Q_{N-K} \in O$. For the definition and existence of affine connections, see [33, [38, 45]. The cocycle is defined by zero on elements of the same type (vector fields or functions) and by

$$
\gamma_{C, T}^{(m)}(e, g):=-\gamma_{C, T}^{(m)}(g, e):=\frac{1}{2 \pi \mathrm{i}} \int_{C}\left(\tilde{e} \cdot g^{\prime \prime}+T \cdot\left(\tilde{e} \cdot g^{\prime}\right)\right) d z
$$

for $e \in \mathcal{L}$ and $g \in \mathcal{A}$. Again, the cohomology class does not depend on the chosen affine connection.

Now we consider cocycles obtained by integration over a separating cycle $C_{S}$. Instead of $\gamma_{C_{S}}$ we will use the symbol $\gamma_{S}$. Clearly, these cocycles can be expressed via residues at the points in $I$ or equivalently at the points in $O$.

Proposition 2.6. 33 The above cocycles (2.16), (2.19) and (2.20) if integrated over a separating cycle $C_{S}$ are local. More precisely, there exist constants $T_{1}, T_{2}, T_{3}$ such that 
for all $m, n \in \mathbb{Z}$

$$
\begin{aligned}
\gamma_{S}^{(f)}\left(\mathcal{A}_{n}, \mathcal{A}_{m}\right) \neq 0 & \Longrightarrow T_{1} \leq m+n \leq 0, \\
\gamma_{S, R}^{(v)}\left(\mathcal{L}_{n}, \mathcal{L}_{m}\right) \neq 0 & \Longrightarrow T_{2} \leq m+n \leq 0, \\
\gamma_{S, T}^{(m)}\left(\mathcal{L}_{n}, \mathcal{A}_{m}\right) \neq 0 & \Longrightarrow T_{3} \leq m+n \leq 0 .
\end{aligned}
$$

If we replace $R$ or $T$ by other meromorphic connections which have poles only at $A$, the cocycle still will be local.

One of the main results of 38 is

Theorem 2.7. (a) Every local cocycle of $\mathcal{A}$ which is $\mathcal{L}$-invariant is multiplicative and vice versa. It is given as a multiple of the cocycle $\gamma_{S}^{(f)}$. The cocycle $\gamma_{S}^{(f)}$ is cohomologically non-trivial.

(b) Every local cocycle of $\mathcal{L}$ is cohomologous to a scalar multiple of $\gamma_{S, R}^{(v)}$. The cocycle $\gamma_{S, R}^{(v)}$ defines a non-trivial cohomology class. For every cohomologically non-trivial local cocycle a meromorphic projective connection $R^{\prime}$ which is holomorphic outside of $A$ can be chosen such that the cocycle is equal to a scalar multiple of $\gamma_{S, R^{\prime}}^{(v)}$.

(c) Every local cocycle $\gamma$ for $\mathcal{D}^{1}$ is a linear combination of the above introduced cocycles $\gamma_{S}^{(f)}, \gamma_{S, R}^{(v)}$, and $\gamma_{S, T}^{(m)}$ up to coboundary, i.e. there exists $r_{1}, r_{2}, r_{3} \in \mathbb{C}$ with

$$
\gamma=r_{1} \gamma_{S}^{(f)}+r_{2} \gamma_{S, T}^{(m)}+r_{3} \gamma_{S, R}^{(v)}+\text { coboundary. }
$$

The 3 basic cocycles are linearly independent in the cohomology space. If the scalars $r_{2}$ and $r_{3}$ in the linear combination are non-zero, then a meromorphic projective connection $R^{\prime}$ and an affine connections $T^{\prime}$, both holomorphic outside $A$, can be found such that $\gamma=r_{1} \gamma_{S}^{(f)}+r_{2} \gamma_{S, T^{\prime}}^{(m)}+r_{3} \gamma_{S, R^{\prime}}^{(v)}$

Multiplying the cocycle with a nonzero scalar corresponds to rescaling the central element. This is an isomorphism of the central extension.

Corollary 2.8. With respect to the almost-grading up to equivalence and rescaling (e.g. up to isomorphy) there exist

(a) a unique almost-graded nontrivial central extension $\widehat{\mathcal{A}}$ of $\mathcal{A}$ which is defined by an $\mathcal{L}$-invariant cocycle,

(b) a unique almost-graded nontrivial central extension $\widehat{\mathcal{L}}$ of $\mathcal{L}$.

These central extensions are given by the above geometric cocycles.

For $\mathcal{D}^{1}$ one obtains up to equivalence a three-dimensional family of one-dimensional central extensions, or equivalently, a three-dimensional central extension.

Remark. In the context of semi-infinite wedge representations associated to the module $\mathcal{F}^{\lambda}$ (see [33], 35], 39]) as cocycle the linear combination

$$
\gamma_{\lambda}=-\left(\gamma_{S}^{(f)}+\frac{1-2 \lambda}{2} \gamma_{S, T_{\lambda}}^{(m)}+2\left(6 \lambda^{2}-6 \lambda+1\right) \gamma_{S, R_{\lambda}}^{(v)}\right),
$$


with a suitable meromorphic affine connection $T_{\lambda}$ and a projective connection $R_{\lambda}$ without poles outside of $A$ and at most poles of order one at the points in $I$ for $T_{\lambda}$ and poles of order two for $R_{\lambda}$ appears. It depends on $\lambda$ and defines a central extension $\widehat{\mathcal{D}}^{1}{ }_{\lambda}$ which has a representation on the space of semi-infinite wedge forms. The cocycle and the action can be extended to a central extension $\widehat{\mathcal{D}}_{\lambda}$ of the whole differential operator algebra.

\section{Affine Algebras}

In this section I introduce the higher genus analogue of the current algebras and their central extensions, the affine algebras (i.e. the Kac-Moody algebras of untwisted affine type). The current algebras come with an almost-grading and one of the main goals of this article is to determine their almost-graded central extensions. Almost-graded central extensions are also called local central extensions.

3.1. Higher genus current algebras. Let $\mathfrak{g}$ be an arbitrary finite-dimensional Lie algebra. The multi-point higher genus current algebra (or multi-point higher genus loop algebra) is defined as

$$
\overline{\mathfrak{g}}:=\mathfrak{g} \otimes \mathcal{A}, \quad \text { with Lie product } \quad[x \otimes f, y \otimes g]:=[x, y] \otimes(f \cdot g) .
$$

We introduce a grading in $\overline{\mathfrak{g}}$ by defining

$$
\operatorname{deg}\left(x \otimes A_{n, p}\right):=n, \quad n \in \mathbb{Z}, p=1, \ldots, K .
$$

Here $A_{n, p}$ are the basis elements (2.10) of the algebra $\mathcal{A}$. This makes $\overline{\mathfrak{g}}$ to an almostgraded Lie algebra, $\overline{\mathfrak{g}}=\oplus_{n \in \mathbb{Z}}(\overline{\mathfrak{g}})_{n}$, with homogenous subspaces $(\overline{\mathfrak{g}})_{m}=\mathfrak{g} \otimes \mathcal{A}_{m}$. To simplify the notation I will sometimes use $x(g)$ to denote the element $x \otimes g$.

The algebra $\mathfrak{g}$ can be embedded into the algebra $\overline{\mathfrak{g}}$ as $\mathfrak{g} \otimes 1$. More precisely,

Lemma 3.1. $1=\sum_{p=1}^{K} A_{0, p}$. In particular, $\mathfrak{g}$ is a subalgebra of $(\overline{\mathfrak{g}})_{0}$.

The lemma follows from the calculation of the basis coefficients of the function 1 , using the Krichever-Novikov duality (2.4). See [41, Lemma 2.6] for details.

A Lie algebra $\mathfrak{g}$ fulfilling $\mathfrak{g}^{\prime}:=[\mathfrak{g}, \mathfrak{g}]=\mathfrak{g}$ is called perfect. Further down we will need the following simple observation.

Proposition 3.2. If $\mathfrak{g}$ is a perfect Lie algebra then $\overline{\mathfrak{g}}$ is also perfect.

Proof. The elements of the type $x \otimes f$ with $x \in \mathfrak{g}$ and $f \in \mathcal{A}$ generate $\overline{\mathfrak{g}}$. Take any $x \otimes g$, then by perfectness of $\mathfrak{g}$ there exists $z, y \in \mathfrak{g}$ with $x=[z, y]$. Hence $x \otimes f=[z \otimes f, y \otimes 1]$. 
3.2. Central extensions. Next we consider central extensions. As explained in Section 2.3 central extensions are given via Lie algebra 2-cocycles with values in the trivial module. For short they will just be called cocycles.

Examples of central extensions are obtained as follows. Let $\alpha$ be a fixed invariant, symmetric bilinear form for the Lie algebra $\mathfrak{g}$, i.e. a form obeying $\alpha([x, y], z)=$ $\alpha(x,[y, z])$. We do not require it to be non-degenerate. If $\mathfrak{g}$ is abelian, every symmetric bilinear form will be invariant and will do. If $\mathfrak{g}$ is a simple Lie algebra any multiple of the Cartan-Killing form will do. Choose a multiplicative cocycle $\gamma$ (see (2.18) for the definition) for $\mathcal{A}$. We set $\widehat{\mathfrak{g}}_{\alpha, \gamma}=\mathbb{C} \oplus \overline{\mathfrak{g}}$ as vector space and introduce a bracket

$$
[\widehat{x \otimes f}, \widehat{y \otimes g}]=[x, \widehat{y] \otimes(f g})+\alpha(x, y) \gamma(f, g) \cdot t, \quad\left[t, \widehat{\mathfrak{g}}_{\alpha, \gamma}\right]=0,
$$

As usual we set $\widehat{x \otimes f}:=(0, x \otimes f)$ and $t:=(1,0)$.

Proposition 3.3. The vector space $\widehat{\mathfrak{g}}_{\alpha, \gamma}$ with structure (3.3) is a Lie algebra and a central extension of $\overline{\mathfrak{g}}$.

Proof. By general constructions of central extensions (see Section 2.3) it is enough to show that $\psi(x \otimes g, y \otimes h):=\alpha(x, y) \gamma(g, h)$ is a cocycle for $\overline{\mathfrak{g}}$. The antisymmetry is clear. Now

$$
\psi([x \otimes f, y \otimes g], z \otimes h)=\psi([x, y] \otimes f g, z \otimes h)=\alpha([x, y], z) \gamma(f g, h) .
$$

By cyclically permuting and adding up the three terms obtained we get

$$
\begin{gathered}
\alpha([x, y], z) \gamma(f g, h)+\alpha([y, z], x) \gamma(g h, f)+\alpha([z, x], y) \gamma(h f, g)= \\
\alpha([x, y], z) \cdot(\gamma(f g, h)+\gamma(g h, f)+\gamma(h f, g))=0
\end{gathered}
$$

Here I used the invariance of $\alpha$ and the multiplicative property of $\gamma$.

Let $C$ be any nonsingular curve on $M$ then $\gamma_{C}(f, g)=\frac{1}{2 \pi \mathrm{i}} \int_{C} f d g$ is a multiplicative cocycle for $\mathcal{A}$ [38. Hence it defines a central extension $\widehat{\mathfrak{g}}_{\alpha, C}$ of $\overline{\mathfrak{g}}$.

3.3. Local cocycles. Of special importance are cocycles which are local (see Definition 2.5.). As explained there, in this case the almost-grading of $\overline{\mathfrak{g}}$ can be extended to the central extension defined via this cocycle by setting for the central element $t$, $\operatorname{deg} t=0$. From Theorem 2.7 we immediately get

Proposition 3.4. Assume that $\alpha \not \equiv 0$, then the cocycle $\alpha(x, y) \gamma_{C}(f, g)$ is local if and only if the integration cycle $C$ is a separating cycle $C_{S}$.

In this case $\widehat{\mathfrak{g}}_{\alpha, S}:=\widehat{\mathfrak{g}}_{\alpha, C_{S}}$ is an almost-graded central extension of $\overline{\mathfrak{g}}$.

It should be remarked that everything depends on the bilinear form $\alpha$. If $\mathfrak{g}$ is simple there is up to multiplication with a scalar only one such form, the Cartan-Killing form. In this case it is even a non-degenerate form.

The algebras obtained via a cocycle $\gamma_{C_{S}}$ (or more general via $\gamma_{C}$ ) are called the higher genus (multi-point) affine Lie algebras (or Krichever-Novikov algebras of affine type). In the classical situation (and $\mathfrak{g}$ simple) these are nothing else then the usual affine 
Lie algebras (i.e. the untwisted affine Kac-Moody algebras). For higher genus and for the two point situations such algebras were introduced by Krichever and Novikov [22, 23, 24, and studied in more detail by Sheinman [42, 43, and by the author for the multi-point situation 35, 36.

Proposition 3.5. Let $\mathfrak{g}$ be a finite-dimensional Lie algebra which fulfills the condition $\mathfrak{g}^{\prime}:=[\mathfrak{g}, \mathfrak{g}] \neq 0$. Let $\gamma$ be a local cocycle of $\overline{\mathfrak{g}}$. Assume that there is an invariant symmetric bilinear form $\alpha$ on $\mathfrak{g}$ fulfilling $\alpha\left(\mathfrak{g}^{\prime}, \mathfrak{g}\right) \neq 0$, and a bilinear form $\gamma^{(f)}$ on $\mathcal{A}$ such that $\gamma$ can be written as

$$
\gamma(x \otimes f, y \otimes z)=\alpha(x, y) \gamma^{(f)}(f, g),
$$

then $\gamma^{(f)}$ is a multiple of the local cocycle $\gamma_{S}^{(f)}$ for the function algebra. In particular this is the case if $\alpha$ nondegenerate.

Proof. First, $\gamma^{(f)}$ is obviously antisymmetric and hence a cocycle for $\mathcal{A}$. We calculate

$$
\gamma([x \otimes f, y \otimes g], z \otimes h)=\gamma([x, y] \otimes f \cdot g, z \otimes h)=\alpha([x, y], z) \gamma^{(f)}(f \cdot g, h) .
$$

For the cocycle condition for the elements $x \otimes f, y \otimes g$ and $z \otimes h$ we have to permute this cyclically and add up the results. We obtain (using the invariance of $\alpha$ )

$$
\alpha([x, y], z)\left(\gamma^{(f)}(f \cdot g, h)+\gamma^{(f)}(g \cdot h, f)+\gamma^{(f)}(h \cdot f, g)=0 .\right.
$$

By the condition $[\mathfrak{g}, \mathfrak{g}] \neq 0$ and by $\alpha\left(\mathfrak{g}^{\prime}, \mathfrak{g}\right) \neq 0$ it follows that we can find $x, y, z \in \mathfrak{g}$ such that $\alpha([x, y], z) \neq 0$. This implies that $\gamma^{(f)}$ is a local and multiplicative cocycle. Theorem 2.7 yields the claim. Note that for $\alpha$ nondegenerate, $\alpha\left(\mathfrak{g}^{\prime}, \mathfrak{g}\right) \neq 0$ is always true.

Proposition 3.6. Let $\gamma$ be a cocycle of $\overline{\mathfrak{g}}$ which can be written as product

$$
\gamma(x \otimes f, y \otimes z)=\alpha(x, y) \cdot \gamma^{(f)}(f, g),
$$

with $\gamma^{(f)}$ a non-vanishing multiplicative cocycle for $\mathcal{A}$ and $\alpha$ a bilinear form on $\mathfrak{g}$ then $\alpha$ is a symmetric invariant bilinear form.

Proof. First we apply the multiplicative property for $\gamma^{(f)}$ to the triple $(1,1, f)$ and obtain $\gamma^{(f)}(1 \cdot 1, f)+\gamma^{(f)}(1 \cdot f, 1)+\gamma^{(f)}(f \cdot 1,1)=0$. This implies that $\gamma^{(f)}(1, f)=0$ for all $f \in \mathcal{A}$. From the antisymmetry of $\gamma$ and $\gamma^{(f)}$ it follows that $\alpha$ is symmetric. Now take $f, h \in \mathcal{A}$ such that $\gamma^{(f)}(f, h) \neq 0$. We obtain

$$
\gamma([x \otimes f, y \otimes 1], z \otimes h)=\alpha([x, y], z) \cdot \gamma^{(f)}(f \cdot 1, h) .
$$

Adding this cyclically permuted up, yields

$$
0=\alpha([x, y], z) \cdot \gamma^{(f)}(f, h)+\alpha([y, z], x) \cdot \gamma^{(f)}(h, f)+\alpha([z, x], y) \cdot \gamma^{(f)}(h f, 1) .
$$

Hence $(\alpha([x, y], z)-\alpha([y, z], x)) \gamma^{(f)}(f, h)=0$. From $\gamma^{(f)}(f, h) \neq 0$ and the symmetry of $\alpha$ the invariance $\alpha([x, y], z)=\alpha(x,[y, z])$ follows. 
A cohomology class containing a representing cocycle which is local is called a local cohomology class. Note that not all elements in a local cohomology class will be local. But the sum of two local cocycles will be local again. The subspace of $\mathrm{H}^{2}(\overline{\mathfrak{g}}, \mathbb{C})$ consisting of local cohomology classes is denoted by $\mathrm{H}_{l o c}^{2}(\overline{\mathfrak{g}}, \mathbb{C})$.

3.4. $\mathcal{L}$-invariant cocycles. The following definition generalizes the definition of $\mathcal{L}$ invariance for cocycles of $\mathcal{A}$ to cocycles of arbitrary current algebras $\overline{\mathfrak{g}}$.

Definition 3.7. A cocycle $\gamma$ of $\overline{\mathfrak{g}}$ is called $\mathcal{L}$-invariant if

$$
\gamma(x(e . g), y(h))+\gamma(x(g), y(e . h))=0, \quad \forall x, y \in \mathfrak{g}, \quad e \in \mathcal{L}, g, h \in \mathcal{A} .
$$

Proposition 3.8. Let $\psi=\delta \phi$ be a coboundary of $\overline{\mathfrak{g}}$ which is local and $\mathcal{L}$-invariant then $\psi=0$.

Proof. The coboundary $\psi$ is given as $\psi(x(g), y(h))=\phi([x, y](g h))$ with a linear form $\phi: \overline{\mathfrak{g}} \rightarrow \mathbb{C}$. By the $\mathcal{L}$-invariance

$$
0=\psi(x(e \cdot g), y(h))+\psi(x(g), y(e \cdot h))=\phi([x, y](e \cdot(g h)) .
$$

We set $h=1$ and obtain

$$
\phi([x, y](e . g))=0, \quad \forall x, y \in \mathfrak{g}, \quad e \in \mathcal{L}, g \in \mathcal{A} .
$$

Assume that $\psi \neq 0$ then there must exist $x, y \in \mathfrak{g}$ and $f \in \mathcal{A}$ such that $\phi([x, y](f)) \neq 0$. Hence, there exists also a basis element $A_{k, r}$ such that $\phi\left([x, y]\left(A_{k . r}\right)\right) \neq 0$. Assume first that $k>0$ then using the almost-graded structure (see Proposition 2.4) for the module $\mathcal{A}$ over $\mathcal{L}$ employed for the pairs of elements $\left(e_{0, r}, A_{k, r}\right)$ we can write (with a certain $S^{\prime} \in \mathbb{N}$ and coefficients $a_{h, s}^{\prime} \in \mathbb{C}$ )

$$
e_{0, r} \cdot A_{k, r}=k A_{k, r}+\sum_{h=k+1}^{k+S^{\prime}} \sum_{s=1}^{K} a_{h, s}^{\prime} A_{h, s} .
$$

The appearing $A_{h, s}$ in the sum can be replaced by $\left(e_{0, s} \cdot A_{h, s}\right) / h+h . d . t$. until we can write

$$
e_{0, r} \cdot A_{k, r}=k A_{k, r}+\sum_{h=k+1}^{S} \sum_{s=1}^{K} a_{h, s}\left(e_{0, s} \cdot A_{h, s}\right)+D, \quad \text { with } D \in \bigoplus_{h>S}^{\infty} \mathcal{A}_{h},
$$

and $S$ chosen such that $\psi\left((\overline{\mathfrak{g}})_{n},(\overline{\mathfrak{g}})_{m}\right)=0$ for $n+m>S$. By the assumed locality of $\psi$ such an $S$ exists. Now we calculate

$0=\phi\left([x, y]\left(e_{0, r} . A_{k, r}\right)\right)=k \phi\left([x, y]\left(A_{k, r}\right)\right)+\sum_{h=k+1}^{S} \sum_{s=1}^{K} a_{h, s} \phi\left([x, y]\left(e_{0, s} . A_{h, s}\right)\right)+\phi([x, y](D))$.

The first equation follows from (3.13). On the r.h.s. the second term will vanish by (3.13) too. The 3. term can be written as $\phi([x, y](D))=\psi(x \otimes D, y \otimes 1)$. Using Lemma 3.1 we obtain that the 3 . term will vanish too. Hence $k \cdot \phi\left([x, y]\left(A_{k, r}\right)\right)=0$ in contradiction to the assumption. 
If $k=0$ we take for the first step $e_{-1, r} \cdot A_{1, r}=1 \cdot A_{0, r}+$ higher terms. For the higher terms we can apply $e_{0, s}$ as above. For $k<0$ we start as above and use instead of $e_{0, s}$. $A_{0, s}$ the element $e_{-1, s} . A_{1, s}$ to obtain $A_{0, s}$ in the corresponding intermediate step. Altogether this leads to a contradiction.

Proposition 3.9. Let $\gamma$ be an $\mathcal{L}$-invariant and local cocycle for the current algebra $\overline{\mathfrak{g}}$ then

$$
\gamma(x, y(g))=0, \quad \forall x, y \in \mathfrak{g}, g \in \mathcal{A} .
$$

Proof. We use the $\mathcal{L}$-invariance (3.12) for $e \in \mathcal{L}, g=1$ and $h \in \mathcal{A}$. It follows $\gamma(x, y(e . h))=0$ for all $e \in \mathcal{L}$ and $h \in \mathcal{A}$. Using the same kind of arguments as in the proof of Proposition 3.8, from the locality of $\gamma$ and the almost-graded structure the claim follows.

We call a cohomology class which has an $\mathcal{L}$-invariant cocycle as an representing element an $\mathcal{L}$-invariant cohomology class. By $\mathrm{H}_{\text {loc, } \mathcal{L}}^{2}(\overline{\mathfrak{g}}, \mathbb{C})$ we denote the subspace of $\mathcal{L}$-invariant and local cocycle classes.

Proposition 3.10. Each class in $\mathrm{H}_{\text {loc, }}^{2}(\overline{\mathfrak{g}}, \mathbb{C})$ contains a unique representing cocycle which is local and $\mathcal{L}$-invariant.

Proof. Let $\gamma_{1}$ and $\gamma_{2}$ be two local and $\mathcal{L}$-invariant cocycles representing the same class. The difference $\gamma_{1}-\gamma_{2}$ is a coboundary which is also local and $\mathcal{L}$-invariant. Proposition 3.8 shows that it has to vanish.

3.5. Current algebras of reductive Lie algebras. Let $\mathfrak{g}$ be a finite-dimensional reductive Lie algebra. Recall (e.g. [5, I.6.4]) that $\mathfrak{g}$ is reductive if and only if $\mathfrak{g}$ is the direct sum of Lie algebras $\mathfrak{g}_{0}, \mathfrak{g}_{1}, \ldots, \mathfrak{g}_{M}$ with $\mathfrak{g}_{0}$ abelian and $\mathfrak{g}_{1}, \ldots, \mathfrak{g}_{M}$ simple Lie algebras, i.e.

$$
\mathfrak{g}=\mathfrak{g}_{0} \oplus \mathfrak{g}_{1} \oplus \cdots \oplus \mathfrak{g}_{M}
$$

In particular, $\mathfrak{g}_{0}$ is the center of $\mathfrak{g}$, for the derived algebra we obtain $\mathfrak{g}^{\prime}=[\mathfrak{g}, \mathfrak{g}]=$ $\mathfrak{g}_{1} \oplus \cdots \oplus \mathfrak{g}_{M}$, and we have $\left[\mathfrak{g}_{i}, \mathfrak{g}_{i}\right]=\mathfrak{g}_{i}$, and $\left[\mathfrak{g}_{i}, \mathfrak{g}_{j}\right]=0$ for $i \neq j$.

For the current algebra $\overline{\mathfrak{g}}=\mathfrak{g} \otimes \mathcal{A}$ we obtain the direct decomposition

$$
\overline{\mathfrak{g}}=\overline{\mathfrak{g}}_{0} \oplus \overline{\mathfrak{g}}_{1} \oplus \cdots \oplus \overline{\mathfrak{g}}_{M}, \quad \text { with } \overline{\mathfrak{g}}_{i}=\mathfrak{g}_{i} \otimes \mathcal{A} .
$$

Furthermore, note that every simple Lie algebra is perfect. Hence using Proposition 3.2 we obtain

$$
\left[\overline{\mathfrak{g}}_{i}, \overline{\mathfrak{g}}_{i}\right]=\overline{\mathfrak{g}}_{i}, \quad\left[\overline{\mathfrak{g}}_{i}, \overline{\mathfrak{g}}_{j}\right]=0, i \neq j, \quad \overline{\mathfrak{g}}^{\prime}=[\overline{\mathfrak{g}}, \overline{\mathfrak{g}}]=\overline{\mathfrak{g}^{\prime}}=\overline{\mathfrak{g}}_{1} \oplus \cdots \oplus \overline{\mathfrak{g}}_{M}
$$

Let $\gamma$ be a cocycle for $\overline{\mathfrak{g}}$ then by restriction $\gamma_{i}:=\gamma_{\mid \overline{\mathfrak{g}}_{i} \times \overline{\mathfrak{g}}_{i}}$ defines a cocycle for the subalgebra and direct summand $\overline{\mathfrak{g}}_{i}$ for $i=0,1, \ldots, M$. Vice versa, given a cocycle $\gamma_{i}$ on $\overline{\mathfrak{g}}_{i}$ we define the extension $\tilde{\gamma}_{i}$ to $\overline{\mathfrak{g}} \times \overline{\mathfrak{g}}$ as $\gamma_{i}$ on $\overline{\mathfrak{g}}_{i} \times \overline{\mathfrak{g}}_{i}$ and zero otherwise. 
Lemma 3.11. (a) $\tilde{\gamma}_{i}$ is a cocycle for $\overline{\mathfrak{g}}$ if and only if $\gamma_{i}$ is a cocycle for $\overline{\mathfrak{g}}_{i}$.

(b) $\tilde{\gamma}_{i}$ is a coboundary for $\overline{\mathfrak{g}}$ if and only if $\gamma_{i}$ is a coboundary for $\overline{\mathfrak{g}}_{i}$.

(c) Let $\gamma$ be a cocycle for $\overline{\mathfrak{g}}$ then

$$
\gamma\left(x_{i}, y_{j}\right)=0, \quad \text { for } x_{i} \in \overline{\mathfrak{g}}_{i}, y_{j} \in \overline{\mathfrak{g}}_{j}, \text { and } i \neq j .
$$

Proof. (a) One direction was already explained above. Assume that $\gamma_{i}$ is a cocycle for $\overline{\mathfrak{g}}_{i}$. Clearly, $\tilde{\gamma}_{i}$ is an antisymmetric bilinear form for $\overline{\mathfrak{g}}$. We have to show the cocycle condition. Let $x=\sum_{j=0}^{M} x_{j}, y=\sum_{k=0}^{M} y_{k}$, and $z=\sum_{l=0}^{M} z_{l}$ be three elements of $\overline{\mathfrak{g}}$ with its unique decomposition with $x_{m}, y_{m}, z_{m} \in \overline{\mathfrak{g}}_{m}$ for $m=0, \ldots, M$. Using (3.19) we obtain

$$
\begin{aligned}
\tilde{\gamma}_{i}([x, y], z)=\tilde{\gamma}_{i}\left(\left[\sum_{j=0}^{M} x_{j},\right.\right. & \left.\left.\sum_{k=0}^{M} y_{k}\right], \sum_{l=0}^{M} z_{l}\right)= \\
& \sum_{j, k, l} \tilde{\gamma}_{i}\left(\left[x_{j}, y_{k}\right], z_{l}\right)=\sum_{j, l} \tilde{\gamma}_{i}\left(\left[x_{j}, y_{j}\right], z_{l}\right)=\gamma_{i}\left(\left[x_{i}, y_{i}\right], z_{i}\right) .
\end{aligned}
$$

Hence from the cocycle condition of $\gamma_{i}$ the cocycle condition for $\tilde{\gamma}_{i}$ follows.

(b) Let $\gamma$ be a coboundary for $\overline{\mathfrak{g}}$, i.e. $\gamma(x, y)=\delta \phi(x, y)=\phi([x, y])$ with $\phi: \overline{\mathfrak{g}} \rightarrow \mathbb{C}$ a linear form. Set $\phi_{i}=\phi_{\mid \overline{\mathfrak{g}}_{i}}$. For $x_{i}, y_{i} \in \overline{\mathfrak{g}}_{i}$ we obtain

$$
\gamma_{i}\left(x_{i}, y_{i}\right)=\gamma\left(x_{i}, y_{i}\right)=\delta \phi\left(x_{i}, y_{i}\right)=\phi\left(\left[x_{i}, y_{i}\right]\right)=\phi_{i}\left(\left[x_{i}, y_{i}\right]\right) \text {. }
$$

Hence $\gamma_{i}$ will be a coboundary for $\overline{\mathfrak{g}}_{i}$. If $\gamma_{i}$ is a coboundary for $\overline{\mathfrak{g}}_{i}$ then

$$
\gamma_{i}\left(x_{i}, y_{i}\right)=\delta \phi_{i}\left(x_{i}, y_{i}\right)=\phi_{i}\left(\left[x_{i}, y_{i}\right]\right)
$$

with a linear form $\phi_{i}$ on $\overline{\mathfrak{g}}_{i}$. We use $\tilde{\phi}_{i}$ to denote $\phi_{i}$ extended by zero on the other components of $\overline{\mathfrak{g}}$. But

$$
\tilde{\gamma}_{i}(x, y)=\gamma_{i}\left(x_{i}, y_{i}\right)=\phi_{i}\left(\left[x_{i}, y_{i}\right]\right)=\tilde{\phi}_{i}([x, y]) .
$$

Hence the claim.

(c) Because $i \neq j$ at least one of the indices is $\neq 0$. Assume $i \neq 0$. Because $\left[\overline{\mathfrak{g}}_{i}, \overline{\mathfrak{g}}_{i}\right]=\overline{\mathfrak{g}}_{i}$ we can write $x_{i}=\left[x_{i}^{(1)}, x_{i}^{(2)}\right]$ with $x_{i}^{(1)}, x_{i}^{(2)} \in \overline{\mathfrak{g}}$. The cocycle condition for $x_{i}^{(1)}, x_{i}^{(2)}$ and $y_{j}$ is

$$
\gamma\left(\left[x_{i}^{(1)}, x_{i}^{(2)}\right], y_{i}\right)+\gamma\left(\left[x_{i}^{(2)}, y_{j}\right], x_{i}^{(1)}\right)+\gamma\left(\left[y_{j}, x_{i}^{(1)}\right], x_{i}^{(2)}\right)=0 .
$$

The last two summands are zero because the commutators are zero. Hence the first summand which equals $\gamma\left(x_{i}, y_{j}\right)$ is also zero.

Proposition 3.12. (a) Let $\gamma$ be a cocycle for $\overline{\mathfrak{g}}, \gamma_{i}=\gamma_{\mid \overline{\mathfrak{g}}_{i} \times \overline{\mathfrak{g}}_{i}}$ its restriction to the $i$-th summand, and $\tilde{\gamma}_{i}$ the extension of $\gamma_{i}$ by zero to $\overline{\mathfrak{g}}$ again as above, then

$$
\gamma=\sum_{i=0}^{M} \tilde{\gamma}_{i}=: \bigoplus_{i=0}^{M} \gamma_{i} .
$$


(b) The above decomposition of $\gamma$ induces

$$
\mathrm{Z}^{2}(\overline{\mathfrak{g}}, \mathbb{C})=\bigoplus_{i=0}^{M} \mathrm{Z}^{2}\left(\overline{\mathfrak{g}}_{i}, \mathbb{C}\right), \quad \mathrm{B}^{2}(\overline{\mathfrak{g}}, \mathbb{C})=\bigoplus_{i=0}^{M} \mathrm{~B}^{2}\left(\overline{\mathfrak{g}}_{i}, \mathbb{C}\right), \quad \mathrm{H}^{2}(\overline{\mathfrak{g}}, \mathbb{C})=\bigoplus_{i=0}^{M} \mathrm{H}^{2}\left(\overline{\mathfrak{g}}_{i}, \mathbb{C}\right),
$$

where $\mathrm{Z}^{2}$ denotes the vector space of 2-cocycles and $\mathrm{B}^{2}$ denotes the vector space of 2coboundaries.

(c) The cocycle $\gamma$ is a local (resp. an $\mathcal{L}$-invariant) cocycle if and only if all $\gamma_{i}, i=$ $0,1, \ldots, M$ are local (resp. $\mathcal{L}$-invariant). In particular,

$$
\mathrm{H}_{l o c}^{2}(\overline{\mathfrak{g}}, \mathbb{C})=\bigoplus_{i=0}^{M} \mathrm{H}_{l o c}^{2}\left(\overline{\mathfrak{g}}_{i}, \mathbb{C}\right), \quad \mathrm{H}_{l o c, \mathcal{L}}^{2}(\overline{\mathfrak{g}}, \mathbb{C})=\bigoplus_{i=0}^{M} \mathrm{H}_{l o c, \mathcal{L}}^{2}\left(\overline{\mathfrak{g}}_{i}, \mathbb{C}\right) .
$$

Proof. Per construction $\gamma$ coincides with $\sum_{i} \tilde{\gamma}_{i}$ on $\overline{\mathfrak{g}}_{j} \times \overline{\mathfrak{g}}_{j}$ for all $j$. By Lemma 3.11 both also coincide on $\overline{\mathfrak{g}}_{j} \times \overline{\mathfrak{g}}_{k}$ for all $j, k$ with $j \neq k$. Hence (a). Part (b) is a reformulation of Lemma 3.11 (a) and (b). Clearly locality and $\mathcal{L}$-invariance is a condition which has to be checked on each component $\overline{\mathfrak{g}}_{i}$ separately. Hence (c) follows.

One way to obtain central extensions of $\overline{\mathfrak{g}}$ was to choose a symmetric invariant bilinear form $\alpha$ for $\mathfrak{g}$ and use the defining equation (3.3). With the same type of argument as above any such $\alpha$ for reductive $\mathfrak{g}$ can be decomposed as

$$
\alpha=\oplus_{i=0}^{M} \alpha_{i}, \quad \text { with } \alpha_{i}=\alpha_{\mid \mathfrak{g}_{i} \times \mathfrak{g}_{i}}
$$

symmetric invariant bilinear forms on $\mathfrak{g}_{i}$. Vice versa, every such sum of $\alpha_{i}$ gives a symmetric invariant bilinear form $\alpha$ on $\mathfrak{g}$. Note also that $\alpha\left(\mathfrak{g}_{i}, \mathfrak{g}_{j}\right)=0$ for $i \neq j$. Recall that for a simple Lie algebra $\mathfrak{g}_{i}$ over $\mathbb{C}$ there is up to multiplication with a scalar $r_{i}$ only one such invariant form, the Cartan-Killing form $\beta_{i}$ of $\mathfrak{g}_{i}$. The form $\beta_{i}$ is non-degenerate. We obtain $\alpha=\alpha_{0}+\sum_{i=1}^{M} r_{i} \beta_{i}$ with $r_{i} \in \mathbb{C}$. For the abelian Lie algebra $\mathfrak{g}_{0}$ every symmetric bilinear form is invariant. If $\operatorname{dim} \mathfrak{g}_{0}=n$ then the space of symmetric bilinear forms on $\mathfrak{g}_{0}$ is $\frac{n(n+1)}{2}$-dimensional. Altogether we obtain that the space of symmetric invariant bilinear forms on $\mathfrak{g}$ is $\frac{n(n+1)}{2}+M$-dimensional.

Our aim is to determine all local (and $\mathcal{L}$-invariant) cocycles for the current algebra $\overline{\mathfrak{g}}$ of a reductive Lie algebra $\mathfrak{g}$. To reach this goal we first have to study the cocycles for the current algebras of the simple, semisimple, and abelian summands of $\mathfrak{g}$.

\subsection{Cocycles for the simple case.}

Theorem 3.13. (a) Let $\mathfrak{g}$ be a finite-dimensional simple Lie algebra, then every local cocycle of the current algebra $\overline{\mathfrak{g}}=\mathfrak{g} \otimes \mathcal{A}$ is cohomologous to a cocycle given by

$$
\gamma(x \otimes f, y \otimes g)=r \cdot \frac{\beta(x, y)}{2 \pi \mathrm{i}} \int_{C_{S}} f d g, \quad \text { with } r \in \mathbb{C},
$$


and $\beta$ the Cartan-Killing form of $\mathfrak{g}$. In particular, $\gamma$ is cohomologous to a local and $\mathcal{L}$-invariant cocycle.

(b) If the cocycle is already local and $\mathcal{L}$-invariant, then it coincides with the cocycle (3.30) with $r \in \mathbb{C}$ suitable chosen.

Proof. Kassel [19] proved that the algebra $\overline{\mathfrak{g}}=\mathfrak{g} \otimes \mathcal{A}$ for any commutative algebra $\mathcal{A}$ over $\mathbb{C}$ and any simple Lie algebra $\mathfrak{g}$ admits a universal central extension. It is given by

$$
\widehat{\mathfrak{g}}^{\text {univ }}=\left(\Omega_{\mathcal{A}}^{1} / d \mathcal{A}\right) \oplus \overline{\mathfrak{g}}
$$

with Lie structure

$$
[x \otimes f, y \otimes g]=[x, y] \otimes f g+\beta(x, y) \overline{f d g}, \quad\left[\Omega_{\mathcal{A}}^{1} / d \mathcal{A}, \widehat{\mathfrak{g}}^{\text {univ }}\right]=0 .
$$

Here $\Omega_{\mathcal{A}}^{1} / d \mathcal{A}$ denotes the vector space of Kähler differentials of the algebra $\mathcal{A}$, and $\beta$ is the Cartan-Killing form. The elements in $\Omega_{\mathcal{A}}^{1}$ can be given as $f d g$ with $f, g \in \mathcal{A}$, and $\overline{f d g}$ denotes its class modulo $d \mathcal{A}$. This universal extension is not necessarily onedimensional. Let $\widehat{\mathfrak{g}}$ be any one-dimensional central extension of $\overline{\mathfrak{g}}$. It will be given as a quotient of $\widehat{\mathfrak{g}}^{\text {univ }}$. Up to equivalence it can be given by a Lie homomorphism $\Phi$

$$
\widehat{\mathfrak{g}}^{\text {univ }}=\Omega_{\mathcal{A}}^{1} / d \mathcal{A} \oplus \overline{\mathfrak{g}} \stackrel{\Phi=(\varphi, i d)}{\longrightarrow} \widehat{\mathfrak{g}}=\mathbb{C} \oplus \overline{\mathfrak{g}}
$$

with a linear form $\varphi$ on $\Omega_{\mathcal{A}}^{1} / d \mathcal{A}$. The structure of $\widehat{\mathfrak{g}}$ is then equal to

$$
[x \otimes f, y \otimes g]=[x, y] \otimes f g+\beta(x, y) \varphi(\overline{f d g}) t, \quad[t, \widehat{\mathfrak{g}}]=0 .
$$

In our situation $M \backslash A$ is an affine curve and $\Omega_{\mathcal{A}}^{1} / d \mathcal{A}$ is the first cohomology group of the complex of meromorphic functions on $M$ which are holomorphic on $M \backslash A$ (similar arguments can be found in an article by Bremner [6]). By Grothendieck's algebraic deRham theorem [15, p.453] the cohomology of the complex is isomorphic to the singular cohomology of $M \backslash A$. Such a linear form $\varphi$ can be given by choosing a linear combination of cycle classes in $M \backslash A$ and integrating the differential class $\overline{f d g}$ over this combination. By Theorem 2.7 the locality implies that the combination is a multiple of the separating cocycle. This shows that the given cocycle is cohomologous to (3.30). But this cocycle is local and $\mathcal{L}$-invariant. Hence, (a). By Proposition 3.10 it is the only local and $\mathcal{L}$-invariant cocycle in his class. Hence, (b).

Corollary 3.14. For $\mathfrak{g}$ simple, up to equivalence of extensions and rescaling of the central element there is a unique non-trivial almost-graded central extension $\widehat{\mathfrak{g}}$, its higher genus multi-point current algebra $\overline{\mathfrak{g}}$. It is given by the cocycle (3.30).

Corollary 3.15. For g simple

$$
\mathrm{H}_{l o c}^{2}(\overline{\mathfrak{g}}, \mathbb{C})=\mathrm{H}_{l o c, \mathcal{L}}^{2}(\overline{\mathfrak{g}}, \mathbb{C}) .
$$

This space is one-dimensional. 
3.7. Cocycles for the semisimple case. Let $\mathfrak{g}$ be a semisimple Lie algebra. It can be written as direct sum of simple Lie algebras $\mathfrak{g}_{i}$, i.e. $\mathfrak{g}=\mathfrak{g}_{1} \oplus \cdots \oplus \mathfrak{g}_{M}$.

Theorem 3.16. (a) For every local cocycle $\gamma$ for the current algebra $\overline{\mathfrak{g}}$ of a semisimple Lie algebra $\mathfrak{g}$ there exists a symmetric invariant bilinear form $\alpha$ for $\mathfrak{g}$ such that $\gamma$ is cohomologous to

$$
\gamma_{\alpha, S}^{\prime}(x \otimes f, y \otimes g)=\frac{\alpha(x, y)}{2 \pi \mathrm{i}} \int_{C_{S}} f d g .
$$

In particular, $\gamma$ is cohomologous to a local and $\mathcal{L}$-invariant cocycle.

(b) If the cocycle is already local and $\mathcal{L}$-invariant, then it coincides with the cocycle (3.36).

$$
\operatorname{dim} \mathrm{H}_{l o c}^{2}(\overline{\mathfrak{g}}, \mathbb{C})=\operatorname{dim} \mathrm{H}_{l o c, \mathcal{L}}^{2}(\overline{\mathfrak{g}}, \mathbb{C})=M,
$$

where $M$ is the number of simple summands of $\mathfrak{g}$.

Proof. As explained in Section 3.5 every local cocycle for $\overline{\mathfrak{g}}$ can be decomposed as $\gamma=\oplus_{i=1}^{M} \gamma_{i}$ with $\gamma_{i}$ local cocycles on the simple summands $\gamma_{i}$. Denote by $\beta_{i}$ the CartanKilling form of $\mathfrak{g}_{i}$. By Theorem 3.13 the $\gamma_{i}$ is cohomologous to

$$
\gamma_{i}^{\prime}\left(x_{i} \otimes f, y_{i} \otimes g\right)=r_{i} \cdot \frac{\beta_{i}\left(x_{i}, y_{i}\right)}{2 \pi \mathrm{i}} \int_{C_{S}} f d g, \quad \text { with } r_{i} \in \mathbb{C} .
$$

We set $\alpha:=\sum_{i=1}^{M} r_{i} \beta_{i}$ which is defined for $x=\sum_{j} x_{j}, y=\sum_{k} y_{k}$ by $a(x, y):=$ $\sum_{i=1}^{M} r_{i} \beta_{i}\left(x_{i}, y_{i}\right)$. The form $\alpha$ is a symmetric invariant bilinear form and

$$
\gamma_{\alpha, S}(x \otimes f, y \otimes g)=\frac{\alpha(x, y)}{2 \pi \mathrm{i}} \int_{C_{S}} f d g
$$

is a cocycle which is cohomologous to $\gamma$ (use Proposition 3.12). This shows (a). Part (b) follows from Proposition 3.10, All linear combinations of the Cartan-Killing forms $\beta_{i}$ give the whole (local) cohomology space. Hence (c). This follows also from Proposition 3.12 (b).

Corollary 3.17. The space of equivalence classes of almost-graded central extensions of the current algebra $\overline{\mathfrak{g}}$ of a semisimple Lie algebra $\mathfrak{g}$ is in 1:1 correspondence with the space of symmetric invariant bilinear forms for $\mathfrak{g}$. Its dimension is the number of simple summands of $\mathfrak{g}$.

Remark. In general, the claim of the above theorem is not true for $\mathfrak{g}$ not semisimple. As a nontrivial example take $\mathfrak{g}=\mathfrak{g l}(n)$. Let $\alpha$ be any nonvanishing symmetric invariant bilinear form for $\mathfrak{g l}(n)$. For any antisymmetric bilinear form $\psi$ on $\mathcal{A}$ the form

$$
\gamma(x \otimes f, y \otimes g):=\alpha(x, y) \psi(f, g)
$$


defines a cocycle for $\overline{\mathfrak{g l}}(n)$. But $\psi$ can be chosen to be local without being a geometric cocycle. We have to require the cocycle to be $\mathcal{L}$-invariant, to obtain a statement corresponding to the above theorem at least in the reductive case, see Section 3.9.

3.8. Cocycles for the abelian case. Let $\mathfrak{g}$ be a finite-dimensional abelian Lie algebra. Note that in the abelian case there exists no non-trivial coboundaries. Hence two different cocycles will never be cohomologous. Already in the one-dimensional abelian case, i.e. $\mathfrak{g}=\mathbb{C}, \overline{\mathfrak{g}}=\mathbb{C} \otimes \mathcal{A}=\mathcal{A}$, if one wants to obtain uniqueness results, it is necessary to allow only $\mathcal{L}$-invariant cocycles. If $\operatorname{dim} \mathfrak{g} \geq 2$ an additional phenomena will show up. In contrast to the semisimple case the Lie algebra $\mathfrak{g}$ will have non-trivial central extensions. Those are given by alternating bilinear forms on $\mathfrak{g}$. If we would allow arbitrary central extensions of $\overline{\mathfrak{g}}$ then also central extensions induced by extensions of $\mathfrak{g}$ would show up. It will turn out, that the required $\mathcal{L}$-invariance will exclude cocycles coming from $\mathfrak{g}$.

Lemma 3.18. Let $\gamma$ be a local and $\mathcal{L}$-invariant cocycle for $\overline{\mathfrak{g}}$.

Set $\gamma_{x y}(f, g):=\gamma(x \otimes f, y \otimes g)$ for fixed $x, y \in \mathfrak{g}$. Then there exists $\alpha_{x y} \in \mathbb{C}$ with

$$
\gamma_{x y}(f, g)=\alpha_{x y} \gamma_{S}^{(f)}(f, g) .
$$

Proof. For fixed $x, y$ the form $\gamma_{x y}$ will be a bilinear form on $\mathcal{A}$. The claim follows with the same kind of arguments as in 38 used for the $\mathcal{L}$-invariant local cocycles for the function algebra $\mathcal{A}$ to show that they are multiples of $\gamma_{S}^{(f)}$. Any reference in the proof there to the antisymmetry of the bilinear form giving the cocycle can be avoided. Hence, $\gamma_{x y}$ has to be a multiple of the function algebra cocycle $\gamma_{S}^{(f)}$. In particular the antisymmetry will follow. For the convenience of the reader the details are given in an appendix to this article.

Theorem 3.19. Let $\mathfrak{g}$ be an abelian Lie algebra of dimension $n$. Then

$$
\operatorname{dim} \mathrm{H}_{l o c, \mathcal{L}}^{2}(\overline{\mathfrak{g}}, \mathbb{C})=\frac{n(n+1)}{2},
$$

and the cocycles are given by

$$
\gamma_{\alpha}(x \otimes f, y \otimes g)=\frac{\alpha(x, y)}{2 \pi \mathrm{i}} \int_{C_{S}} f d g
$$

where $\alpha$ is an arbitrary symmetric bilinear form for $\mathfrak{g}$.

Proof. For $n=1$ this is the result for $\overline{\mathfrak{g}}=\mathcal{A}$ obtained in [38] and here recalled in Theorem 2.7. Clearly, given any such $\alpha$ we obtain via (3.42) a cocycle which is local and $\mathcal{L}$-invariant. Now let $\gamma$ be a local and $\mathcal{L}$-invariant cocycle. From Lemma 3.18 we know that we can write $\gamma(x \otimes f, y \otimes g)=\alpha_{x y} \cdot \gamma_{S}^{(f)}$. The map $\alpha:(x, y) \rightarrow \alpha_{x, y}$ is bilinear. By the antisymmetry of $\gamma$, and $\gamma_{S}^{(f)}$, and the non-vanishing of the latter, $\alpha$ has to be a symmetric form on $\mathfrak{g}$. Hence, $\gamma$ is indeed of the form (3.42). But there are exactly 
$\frac{n(n+1)}{2}$ symmetric bilinear forms which are linearly independent. The corresponding $\gamma_{\alpha} \mathrm{S}$ will stay linearly independent. This shows the formula for the dimension.

3.9. Cocycles for the reductive case. Let $\mathfrak{g}=\mathfrak{g}_{0} \oplus \mathfrak{g}_{1} \oplus \cdots \oplus \mathfrak{g}_{M}$ the decomposition of the reductive Lie algebra $\mathfrak{g}$ into its abelian and simple summands.

Theorem 3.20. (a) Given a cocycle $\gamma$ for $\overline{\mathfrak{g}}$ which is local, and whose restriction $\gamma_{0}$ to the abelian summand $\overline{\mathfrak{g}}_{0}$ is $\mathcal{L}$-invariant, then there exists a symmetric invariant bilinear form $\alpha$ for $\mathfrak{g}$ such that $\gamma$ is cohomologous to

$$
\gamma_{\alpha}^{\prime}(x \otimes f, y \otimes g)=\frac{\alpha(x, y)}{2 \pi \mathrm{i}} \int_{C_{S}} f d g .
$$

Vice versa, every such $\alpha$ determines a local and $\mathcal{L}$-invariant cocycle.

(b) If the cocycle $\gamma$ is already local and $\mathcal{L}$-invariant for the whole $\overline{\mathfrak{g}}$, then it coincides with the cocycle $\gamma_{a}^{\prime}$.

(c)

$$
\operatorname{dim} \mathrm{H}_{l o c, \mathcal{L}}^{2}(\overline{\mathfrak{g}}, \mathbb{C})=\frac{n(n+1)}{2}+M
$$

Proof. Again (a) is obtained by restricting the cocycle to the semisimple and the abelian summand. Using Theorem 3.16 and Theorem 3.19 together with Proposition 3.12 we obtain the results.

Corollary 3.21. Those equivalence classes of almost-graded central extensions of the current algebra $\overline{\mathfrak{g}}$ of a reductive Lie algebra $\mathfrak{g}$, whose corresponding cocycles can be given by $\mathcal{L}$-invariant cocycles, correspond 1:1 to the space of symmetric invariant bilinear forms $\alpha$ on $\mathfrak{g}$. This space has the dimension $\frac{n(n+1)}{2}+M$.

\section{Algebras of Differential operators}

In Section 2 the algebra of differential operators of degree $\leq 1$ associated to the function algebra $\mathcal{A}$ was introduced as semi-direct sum of $\mathcal{A}$ with the vector field algebra $\mathcal{L}$. In this section the construction will be extended to the case when $\mathcal{A}$ is replaced by the current algebra $\overline{\mathfrak{g}}$ of a general finite-dimensional Lie algebra $\mathfrak{g}$. Again, central extensions are studied and classification results for local cocycles are given. The algebras play an important role in the context of Casimir operators in fermionic representations, (e.g. see [44, 45]). The fermionic representations generalize the semi-infinite wedge representations which were studied in [23, 33, 39].

4.1. Differential operator algebras. Let $\mathfrak{g}$ be an arbitrary finite-dimensional Lie algebra, $\overline{\mathfrak{g}}$ the associated current algebra. Set $\mathcal{D}_{\mathfrak{g}}^{1}:=\overline{\mathfrak{g}} \oplus \mathcal{L}$ as vector space. By

$$
e .(x \otimes g):=x \otimes(e . g), \quad e \in \mathcal{L}, x \in \mathfrak{g}, g \in \mathcal{A},
$$

an $\mathcal{L}$-module structure is defined on the space $\overline{\mathfrak{g}}$. 
Definition 4.1. The Lie algebra $\mathcal{D}_{\mathfrak{g}}^{1}$ is the semi-direct sum structure on $\overline{\mathfrak{g}} \oplus \mathcal{L}$ induced by $[e, x \otimes g]:=x \otimes(e \cdot g)$.

In more detail, the product is given as (for $e, f \in \mathcal{L}, x, y \in \mathfrak{g}, g, h \in \mathcal{A}$ )

$$
[(x \otimes g, e),(y \otimes h, f)]=([x, y] \otimes(g \cdot h+g \cdot(e . h)-(f \cdot g) \cdot h),[e, f]) .
$$

There is the short exact sequence of Lie algebras

$$
0 \longrightarrow \overline{\mathfrak{g}} \stackrel{i_{1}}{\longrightarrow} \mathcal{D}_{\mathfrak{g}}^{1} \stackrel{p_{2}}{\longrightarrow} \mathcal{L} \longrightarrow 0 .
$$

Using the almost-gradings of $\overline{\mathfrak{g}}$ and of $\mathcal{L}$ we obtain an almost-grading for $\mathcal{D}_{\mathfrak{g}}^{1}$ by taking $\left(\mathcal{D}_{\mathfrak{g}}^{1}\right)_{m}:=(\overline{\mathfrak{g}})_{m} \oplus \mathcal{L}_{m}$ for $m \in \mathbb{Z}$ as homogenous subspaces. From (4.2) one immediately verifies that this defines an almost-graded structure. For $\mathfrak{g}=\mathbb{C}$ (and hence $\overline{\mathfrak{g}}=\mathcal{A}$ ) we recover the algebra $\mathcal{D}^{1}$ as special case.

4.2. Cocycle conditions. Next we consider cocycles and central extensions of $\mathcal{D}_{\mathfrak{g}}^{1}$. By bilinearity the cocycle condition (2.11) can be equivalently formulated by considering cocycle conditions for triples of elements of "pure types", i.e. elements which are either vector fields or elements from $\overline{\mathfrak{g}}$.

(1) If all three are vector fields, the condition is that the cocycle defines by restriction a cocycle for $\mathcal{L}$.

(2) If all three elements are from $\overline{\mathfrak{g}}$, the condition is that the cocycle defines by restriction a cocycle for $\overline{\mathfrak{g}}$.

(3) Now let $e, f \in \mathcal{L}$ and $x(g) \in \overline{\mathfrak{g}}$, then using the Lie structure we obtain

$$
\gamma([e, f], x(g))-\gamma(e, x(f \cdot g))+\gamma(f, x(e \cdot g))=0 .
$$

(4) If $e \in \mathcal{L}$ and $x(g), y(h) \in \overline{\mathfrak{g}}$ then

$$
\gamma(x(e \cdot g), y(h))-\gamma(e,[x, y](g h))+\gamma(x(g), y(e \cdot h))=0 .
$$

An antisymmetric form on $\overline{\mathfrak{g}}$ will be a cocycle if and only if all 4 conditions are fulfilled.

4.3. Extensions of cocycles. In the following for a cocycle of a subalgebra $V$ of $\mathcal{D}_{\mathfrak{g}}^{1}$ I will use the expression "extended by zero on the complementary space". By this I mean that the extended bilinear form coincides with the cocycle on pairs of elements from $V$ and will be set to zero if any of the two entries in the bilinear form is from the complementary subspace. The rest follows from bilinear extension. Note that this extension is not necessarily a cocycle for $\mathcal{D}_{\mathfrak{g}}^{1}$.

\section{Proposition 4.2.}

(a) Every cocycle of $\mathcal{L}$ can be extended by zero on $\overline{\mathfrak{g}}$ to a cocycle of $\mathcal{D}_{\mathfrak{g}}^{1}$.

(b) A cocycle of $\overline{\mathfrak{g}}$ can be extended by zero on $\mathcal{L}$ to a cocycle of $\mathcal{D}_{\mathfrak{g}}^{1}$ if and only if the cocycle is $\mathcal{L}$-invariant (see Definition 3.7).

(c) Let $\alpha$ be an invariant symmetric bilinear form for $\mathfrak{g}$. The geometric cocycles

$$
\gamma_{\alpha, C}(x(g), y(h))=\alpha(x, y) \cdot \gamma_{C}^{(f)}(g, h)
$$


of $\overline{\mathfrak{g}}$ can be extended by zero to a cocycle for $\mathcal{D}_{\mathfrak{g}}^{1}$.

Proof. From the above 4 separate cocycle conditions only the first one is of relevance for a cocycle of $\mathcal{L}$ which is extended by zero. This is exactly the condition that it is a cocycle for $\mathcal{L}$. This follows also from the sequence (4.3) because the extended cocycle is nothing but the pull-back by $p_{2}$. Hence, (a). The situation is different for cocycles of $\overline{\mathfrak{g}}$. If we set the cocycle to be zero outside of $\overline{\mathfrak{g}}$, then $(1),(2)$, and (3) are automatically fulfilled. From (4.5) it follows that it will define a cocycle if and only if

$$
\gamma(x(e \cdot g), y(h))+\gamma(x(g), y(e \cdot h))=0 .
$$

But this is Definition 3.7 of $\mathcal{L}$-invariance for cocycles of $\overline{\mathfrak{g}}$. Hence, (b). Part (c) follows from the fact that $\gamma_{C}^{(f)}$ is $\mathcal{L}$-invariant, hence the same is true for $\gamma_{\alpha, C}(x(g), y(h))$.

4.4. Cocycles of mixing type. Now we consider cocycles $\gamma$ of pure mixing type. By a cocycle of pure mixing type I mean a cocycle which vanishes on pairs of elements of the same type. From (4.5) it follows that

$$
\gamma(e,[x, y](g))=0, \quad \forall x, y \in \mathfrak{g}, e \in \mathcal{L}, g \in \mathcal{A},
$$

is a necessary condition for $\gamma$ to be a cocycle of pure mixing type.

Proposition 4.3. For a perfect Lie algebra $\mathfrak{g}$ (i.e. $\mathfrak{g}=[\mathfrak{g}, \mathfrak{g}]$ ) there exist no nonvanishing cocycles of $\mathcal{D}_{\mathfrak{g}}^{1}$ of pure mixing type.

Proof. By assumption we can express every $z \in \mathfrak{g}$ as $z=[x, y]$. Hence by (4.8) the claim follows.

Further down we will need the following result.

Lemma 4.4. Let $\gamma$ be a cocycle of pure mixing type which is a coboundary. Then there exists a linear form $\psi: \overline{\mathfrak{g}} \oplus \mathcal{L} \rightarrow \mathbb{C}$ which vanishes on $\mathcal{L}$ with $\gamma=\delta \psi$.

Proof. As a coboundary $\gamma=\delta \psi^{\prime}$ with $\psi^{\prime}: \overline{\mathfrak{g}} \oplus \mathcal{L} \rightarrow \mathbb{C}$ a linear form. We decompose $\psi^{\prime}=\psi_{\mid \overline{\mathfrak{g}}}^{\prime} \oplus \psi_{\mid \mathcal{L}}^{\prime}$. For all $e, f \in \mathcal{L}$ we obtain

$$
0=\gamma(e, f)=\delta \psi(e, f)=\psi([e, f]),
$$

by the requirement of pure mixing type. Hence, $\psi_{\mid[\mathcal{L}, \mathcal{L}]}^{\prime} \equiv 0$. We extend $\psi_{\mid \bar{g}}^{\prime}$ by zero on $\mathcal{L}$ to a linear form $\psi$. Note that $\psi([e, x(g)])=\psi(x(e . g))=\psi^{\prime}([e, x(g)])$ and $\psi([x(g), y(h)])=\psi^{\prime}([x(g), y(h)])=0$. Hence we get $\gamma=\delta \psi$ with a linear form $\psi$ of the required kind.

Let $\gamma$ be a cocycle of pure mixing type which is of the form $\gamma(e, x(g))=\phi(x) \gamma^{(m)}(e, g)$ with $\phi \in \mathfrak{g}^{*}$ a linear form on $\mathfrak{g}$, and $\gamma^{(m)}$ bilinear on $\mathcal{L} \times \mathcal{A}$. Condition (4.8) implies that either $\gamma^{(m)} \equiv 0$ or that $\phi([x, y])=0$ for all $x, y \in \mathfrak{g}$. By Condition (4.4) for $x \in \mathfrak{g}$, $e, f \in \mathcal{L}$ and $g \in \mathcal{A}$ the relation

$$
\phi(x)\left(\gamma^{(m)}([e, f], g)-\gamma^{(m)}(e, f \cdot g)+\gamma^{(m)}(f, e \cdot g)\right)=0
$$


follows. Excluding the trivial case $\phi \equiv 0$ this implies that $\gamma^{(m)}$ is a mixing cocycle for $\mathcal{D}^{1}$. Vice versa, every bilinear form $\gamma(e, x(g))=\phi(x) \gamma^{(m)}(e, g)$ with $\phi \in \mathfrak{g}^{*}$ such that $\phi_{\mid[\mathfrak{g}, \mathfrak{g}]}=0$, and $\gamma^{(m)}$ a mixing cocycle of $\mathcal{D}^{1}$ defines a cocycle of $\mathcal{D}_{\mathfrak{g}}^{1}$.

4.5. The general case. Without assuming further properties of the finite-dimensional Lie algebra $\mathfrak{g}$ we obtain the following theorem.

Theorem 4.5. Let $\mathfrak{g}$ be a finite-dimensional Lie algebra.

(a) Let $\gamma^{(f)}$ be any $\mathcal{L}$-invariant cocycle of the function algebra, $\gamma^{(v)}$ any cocycle of the vector field algebra $\mathcal{L}$, and $\gamma^{(m)}$ any mixing cocycle of $\mathcal{D}^{1}$. Furthermore, let $\alpha$ be any symmetric invariant bilinear form on $\mathfrak{g}$ and $\phi$ any linear form on $\mathfrak{g}$ which vanishes on the derived subalgebra $\mathfrak{g}^{\prime}=[\mathfrak{g}, \mathfrak{g}]$, then

$$
\begin{aligned}
\gamma((x(g), e),(y(h), f)) & =r_{1} \alpha(x, y) \gamma^{(f)}(g, h) \\
& +r_{2}\left(\phi(y) \gamma^{(m)}(e, h)-\phi(x) \gamma^{(m)}(f, g)\right)+r_{3} \gamma^{(v)}(e, f)
\end{aligned}
$$

for arbitrary $r_{1}, r_{2}, r_{3} \in \mathbb{C}$ defines a cocycle of $\mathcal{D}_{\mathfrak{g}}^{1}$.

(b) Let $\gamma$ be a local cocycle of $\mathcal{D}_{\mathfrak{g}}^{1}$. Assume that it can be written as

$$
\begin{aligned}
\gamma((x(g), e),(y(h), f)) & =r_{1} \alpha(x, y) \tilde{\gamma}^{(f)}(g, h) \\
& +r_{2}\left(\phi(y)\left(\tilde{\gamma}^{(m)}(e, h)-\phi(x) \tilde{\gamma}^{(m)}(f, g)\right)+r_{3} \tilde{\gamma}^{(v)}(e, f)\right.
\end{aligned}
$$

with a symmetric invariant bilinear form $\alpha$ for $\mathfrak{g}$ fulfilling $\alpha\left(\mathfrak{g}^{\prime}, \mathfrak{g}\right) \neq 0, \phi$ a linear form on $\mathfrak{g}, \tilde{\gamma}^{(f)}$ a bilinear form on $\mathcal{A}, \tilde{\gamma}^{(v)}$ a bilinear form on $\mathcal{L}$ and $\tilde{\gamma}^{(m)}$ bilinear form on $\mathcal{L} \times \mathcal{A}$, and $r_{1}, r_{2}, r_{3} \in \mathbb{C}$. Assume either $[\mathfrak{g}, \mathfrak{g}] \neq 0$ or that $\tilde{\gamma}^{(f)}$ is $\mathcal{L}$-invariant. Then, ignoring all terms which are identically zero, the remaining forms are multiples of the corresponding unique local cocycles introduced in Section [2.3, respectively are coboundaries, and the linear form $\phi$ vanishes on $\mathfrak{g}^{\prime}=[\mathfrak{g}, \mathfrak{g}]$.

Proof. Part (a) follows from the discussion in Section 4.4 and Section 4.3. To see (b) we note that the restriction of $\gamma$ to $\mathcal{L}$ defines a local cocycle hence for this part Theorem 2.7 shows the correct form. The restriction of the cocycle to $\overline{\mathfrak{g}}$ also defines a cocycle. Hence if $[\mathfrak{g}, \mathfrak{g}] \neq 0$ we can use Proposition 3.5 and obtain the claim for this part. In case $[\mathfrak{g}, \mathfrak{g}]=0$ we assumed $\mathcal{L}$-invariance of $\tilde{\gamma}^{(f)}$ and by locality of the cocycle Theorem 2.7 shows the claim directly. Hence, the first term and the last term define cocycles of $\mathcal{D}_{\mathfrak{g}}^{1}$. This implies that the second term is also a cocycle. In particular, it is a pure mixing cocycle. For the mixing cocycle the relation (4.10) shows that either $\phi$ is identical zero (and the term does not appear at all) or $\tilde{\gamma}^{(m)}$ defines a local cocycle for $\mathcal{D}^{1}$. Again Theorem 2.7 shows the claim. Finally we saw above that for these kind of cocycles the linear form $\phi$ has to vanish on $[\mathfrak{g}, \mathfrak{g}]$.

Note in particular that for $\mathfrak{g}$ semisimple there will be no mixing term in (4.11) and (4.12), see Proposition 4.3. 
4.6. Cocycles for the semisimple case. If $\mathfrak{g}$ is reductive, we will sharpen the results. First we deal with the semisimple case.

Theorem 4.6. (a) Let $\mathfrak{g}$ be a semisimple Lie algebra and $\gamma$ a local cocycle of $\mathcal{D}_{\mathfrak{g}}^{1}$. Then there exists a symmetric invariant bilinear form $\alpha$ for $\mathfrak{g}$ such that $\gamma$ is cohomologous to a linear combination of the local cocycle $\gamma_{\alpha, S}$ given by (3.36) and of the local cocycle $\gamma_{S, R}^{(v)}$ (2.19) for $C=C_{S}$ of the vector field algebra $\mathcal{L}$.

(b) If $\mathfrak{g}$ is a simple Lie algebra, then $\gamma_{\alpha, S}$ is a multiple of the standard cocycle (3.30) for $\mathfrak{g}$.

(c) $\operatorname{dim} \mathrm{H}_{\text {loc }}^{2}\left(\mathcal{D}_{\mathfrak{g}}^{1}, \mathbb{C}\right)=M+1$, where $M$ is the number of simple summands of $\mathfrak{g}$.

Proof. Let $\gamma$ be a local cocycle. By restricting it to $\mathcal{L}$ and $\overline{\mathfrak{g}}$ we obtain local cocycles $\gamma_{1}$ and $\gamma_{2}$. By Theorem 3.16, $\gamma_{2}$ is cohomologous to the cocycle $\gamma_{\alpha, S}$ on $\overline{\mathfrak{g}}$ with a suitable $\alpha$. The necessary coboundary $\delta \phi$ can be extended to the whole algebra by setting the linear form $\phi$ zero on $\mathcal{L}$. Hence by replacing $\gamma$ by a cohomologous cocycle we might even assume that $\gamma_{2}$ is already $\gamma_{\alpha, S}$. But this cocycle can be extended by zero to the whole algebra. Hence, $\gamma-\left(\gamma_{1}+\gamma_{2}\right)$ is again a cocycle which is now of pure mixing type. By Proposition 4.3 for $\mathfrak{g}$ semisimple they are vanishing. This shows (a). Statement (b) follows from the uniqueness of the invariant symmetric bilinear forms for simple Lie algebras. Statement (c) follows from Theorem 3.16.

If the cocycle $\gamma_{2}$ appearing in the proof of Theorem 4.6 restricted to $\overline{\mathfrak{g}}$ is $\mathcal{L}$-invariant then by Theorem 3.16 (b) the cocycle $\gamma_{2}$ equals already $\gamma_{\alpha, S}$.

4.7. Cocycles for the abelian case. Let $\mathfrak{g}$ be an abelian Lie algebra and $\gamma$ a local cocycle for $\mathcal{D}_{\mathfrak{g}}^{1}$. From (4.5) it follows that

$$
\gamma(x(e \cdot g), y(h))+\gamma(x(e \cdot g), y(h))=0 .
$$

By definition this implies that $\gamma_{2}:=\gamma_{\mid \bar{g}}$ is $\mathcal{L}$-invariant. Also $\gamma_{1}:=\gamma_{\mid \mathcal{L}}$ is a vector field cocycle. Both cocycles can be extended by zero on the complement to $\mathcal{D}_{\mathfrak{g}}^{1}$. Hence $\gamma-\left(\gamma_{1}+\gamma_{2}\right)$ will be a local cocycle for $\mathcal{D}_{\mathfrak{g}}^{1}$ which is of pure mixing type.

Proposition 4.7. Every local cocycle $\gamma$ of pure mixing type is cohomologous to

$$
\gamma_{\phi}(e, x(g)):=\frac{\phi(x)}{2 \pi \mathrm{i}} \int_{C_{S}}\left(\tilde{e} \cdot g^{\prime \prime}+T \cdot\left(\tilde{e} \cdot g^{\prime}\right)\right) d z,
$$

with a suitable linear form $\phi \in \mathfrak{g}^{*}$. Vice versa, every $\phi$ defines via (4.14) a local cocycle of pure mixing type.

Proof. If $\operatorname{dim} \mathfrak{g}=1$ then by a result of [38, recalled in Theorem 2.7 $\gamma$ is up to coboundary a scalar multiple of $\gamma_{S, T}^{(m)}$, the standard mixing cocycle, see Equation (2.20). Let $x_{1}, \ldots, x_{n}$ be a basis of $\mathfrak{g}$. Set $\mathcal{A}_{i}:=x_{i} \otimes \mathcal{A} \cong \mathcal{A}$ and $\mathcal{D}_{i}:=\mathcal{A}_{i} \oplus \mathcal{L}$. The space $\mathcal{D}_{i}$ is a subalgebra of $\mathcal{D}_{\mathfrak{g}}^{1}$ isomorphic to $\mathcal{D}^{1}$. Restricting the cocycle to $\mathcal{D}_{i}$ we obtain a cocycle $\gamma_{i}$ 
cohomologous to $r_{i} \gamma_{S, T}^{(m)}$ with suitable $r_{i} \in \mathbb{C}$. We set $\phi\left(x_{i}\right):=r_{i}$ and extend $\phi$ linearly to $\mathfrak{g}$. If $x=\sum_{i} s_{i} x_{i}$ then

$$
\gamma(e, x(g))=\sum_{i} s_{i} \gamma\left(e, x_{i}(g)\right) \sim\left(\sum_{i} s_{i} r_{i}\right) \gamma_{S, T}^{(m)}(e, g)=\phi(x) \gamma_{S, T}^{(m)}(e, g) .
$$

Here $\sim$ denotes cohomologous equivalent. For this step the following remark has to be taken in account. The individual cocycles $\gamma_{i}$ are cohomologous to the standard cocycle. The individual coboundary is determined by a linear form on $\mathcal{A}_{i} \oplus \mathcal{L}$. By the pure mixing type using Lemma 4.4 the corresponding form can by chosen to vanish on the summand $\mathcal{L}$. Hence after such a modification they glue together to a linear form on $\overline{\mathfrak{g}}$ and define a coboundary for $\mathcal{D}_{\gamma}^{1}$. This shows the formula (4.14). Clearly, every such expression is a local cocycle of pure mixing type.

Theorem 4.8. Let $\mathfrak{g}$ be an abelian finite-dimensional Lie algebra. The subspace of local cohomology classes $\mathrm{H}_{\text {loc }}^{2}\left(\mathcal{D}_{\mathfrak{g}}^{1}, \mathbb{C}\right)$ of $\mathrm{H}^{2}\left(\mathcal{D}_{\mathfrak{g}}^{1}, \mathbb{C}\right)$ is $\frac{n(n+1)}{2}+n+1$-dimensional. Up to coboundary every local cocycle is a linear combination of

$$
\gamma_{\alpha}(x(f), y(g))=\frac{\alpha(x, y)}{2 \pi \mathrm{i}} \int_{C_{S}} f d g,
$$

with an arbitrary symmetric bilinear form $\alpha$, of $\gamma_{\phi}$ with an arbitrary linear form $\phi$ (see (4.14)), and of $\gamma_{S, R}^{(v)}($ see (2.19) ).

Proof. Let $\gamma$ be a local cocycle for $\mathcal{D}_{\mathfrak{g}}^{1}$. As explained in the beginning of this subsection such $\gamma$ can be written by restriction as $\gamma=\gamma_{\mid \mathcal{L}}+\gamma_{\mid \bar{g}}+\gamma_{3}$ with $\gamma_{3}$ a local cocycle of pure mixing type. By Proposition 4.7. $\gamma_{3}$ can be given up to coboundary as $\gamma_{\phi}$ with a suitable $\phi$. The cocycle $\gamma_{\mid \mathcal{L}}$ is a local vector field cocycle. Hence, $\gamma_{\mid \mathcal{L}}$ is a multiple of $\gamma_{S, R}^{(v)}$ (see Theorem 2.7). By Theorem 3.19, $\gamma_{\mid \bar{g}}=\gamma_{\alpha}$ with a suitable symmetric bilinear form $\alpha$. Hence every local cocycle is cohomologous to a linear cocycle of the required type. Vice versa, every such linear combination is a local cocycle and all the basis cocycles remain linearly independent.

4.8. Cocycles for the reductive case. Here we have to combine the results on the semisimple and the abelian case. Let $\mathfrak{g}=\mathfrak{g}_{0} \oplus \mathfrak{g}_{1} \oplus \cdots \oplus \mathfrak{g}_{M}$ be the usual decomposition with $\mathfrak{g}_{0}$ abelian of dimension $n$ and the $\mathfrak{g}_{1}, \ldots, \mathfrak{g}_{M}$ simple. Let $\mathfrak{g}^{\prime}=[\mathfrak{g}, \mathfrak{g}]=\mathfrak{g}_{1} \oplus \cdots \oplus \mathfrak{g}_{M}$ be the derived subalgebra, which is now semisimple. The algebras $\mathcal{D}_{\mathfrak{g}_{0}}^{1}$ and $\mathcal{D}_{\mathfrak{g}^{\prime}}^{1}$ are in a natural way subalgebras of $\mathcal{D}_{\mathfrak{g}}^{1}$. They are even ideals, but not complementary ones.

Proposition 4.9. Let $\gamma$ be a cocycle of $\mathcal{D}_{\mathfrak{g}}^{1}$ then $\gamma$ restricted to $\overline{\mathfrak{g}}$ is $\mathcal{L}$-invariant if and only if $\gamma$ restricted to $\overline{\mathfrak{g}}^{\prime}$ is $\mathcal{L}$-invariant.

Proof. We only have to show that a cocycle $\gamma$ of $\mathcal{D}_{\mathfrak{g}}^{1}$ which restricted to $\overline{\mathfrak{g}}^{\prime}$ is $\mathcal{L}$-invariant is also $\mathcal{L}$-invariant if restricted to $\overline{\mathfrak{g}}$. By the cocycle condition (4.5) the $\mathcal{L}$-invariance on $\overline{\mathfrak{g}}$ is true if and only if $\gamma(e,[x, y](g h))=0$ for $x, y \in \mathfrak{g}$. $\mathfrak{g}$ is reductive, hence 
$\mathfrak{g}^{\prime}=\left[\mathfrak{g}^{\prime}, \mathfrak{g}^{\prime}\right]$. This implies $[x, y]=\left[x^{\prime}, y^{\prime}\right]$ with suitable $x^{\prime}, y^{\prime} \in \mathfrak{g}^{\prime}$, and $\gamma\left(e,\left[x^{\prime}, y^{\prime}\right](g h)\right)=0$ by assumption.

Proposition 4.10. (a) Every local cocycle for $\mathcal{D}_{\mathfrak{g}}^{1}$ restricted to $\overline{\mathfrak{g}}_{0}$ is $\mathcal{L}$-invariant.

(b) Every local cocycle for $\mathcal{D}_{\mathfrak{g}}^{1}$ is cohomologous to a local cocycle which restricted to $\mathfrak{g}$ is an $\mathcal{L}$-invariant cocycle.

Proof. (a) Let $\gamma$ be a local cocycle for $\mathcal{D}_{\mathfrak{g}}^{1}$. It defines by restriction to $\mathcal{D}_{\mathfrak{g}_{0}}^{1}$ a local cocycle. Using (4.5) for $\mathcal{D}_{\mathfrak{g}_{0}}^{1}$ we obtain, using $\left[x_{0}, y_{0}\right]=0$, that the restriction to $\overline{\mathfrak{g}}_{0}$ is $\mathcal{L}$-invariant.

(b) Let $\gamma$ be a local cocycle. By Theorem 4.6, $\gamma$ restricted to $\mathcal{D}_{\mathfrak{g}^{\prime}}^{1}$ is cohomologous to a linear combination of the $\mathcal{L}$-invariant cocycle (3.36) and the separating vector field cocycle. Let $\psi=\delta \varphi$ be the coboundary, which appears as difference. The linear form $\varphi$ can be extended to $\tilde{\varphi}$ by setting it zero on $\mathfrak{g}_{0}$. We obtain a coboundary $\tilde{\psi}=\delta \tilde{\varphi}$ on $\mathcal{D}_{\mathfrak{g}}^{1}$. The cocycle $\gamma^{\prime}=\gamma+\tilde{\psi}$ is cohomologous to the one we started with and restricted to $\overline{\mathfrak{g}^{\prime}}$ it is $\mathcal{L}$-invariant. The claim (b) follows now from Proposition 4.9.

Theorem 4.11. (a) For every local cocycle $\gamma$ for $\mathcal{D}_{\mathfrak{g}}^{1}$ there exists a symmetric invariant bilinear form $\alpha$ of $\mathfrak{g}$, and a linear form $\phi$ of $\mathfrak{g}$ which vanishes on $\mathfrak{g}^{\prime}$, such that $\gamma$ is cohomologous to

$$
\gamma^{\prime}=r_{1} \gamma_{\alpha, S}+r_{2} \gamma_{\phi, S}+r_{3} \gamma_{S, R}^{(v)}
$$

with $r_{1}, r_{2}, r_{3} \in \mathbb{C}$, a current algebra cocycle $\gamma_{\alpha, S}$ given by (3.36), a mixing cocycle $\gamma_{\phi, S}$ given by (4.14), and the vector field cocycle $\gamma_{S, R}^{(v)}$ given by (2.19). Vice versa, any such $\alpha, \phi, r_{1}, r_{2}, r_{3} \in \mathbb{C}$ determine a local cocycle.

(b) The space of local cocycles $\mathrm{H}_{\text {loc }}^{2}\left(\mathcal{D}_{\mathfrak{g}}^{1}, \mathbb{C}\right)$ is $\frac{n(n+1)}{2}+n+M+1$ dimensional.

Proof. By Proposition 4.10 we may replace $\gamma$ by a cohomologous cocycle which restricted to $\overline{\mathfrak{g}}$ is $\mathcal{L}$-invariant. We denote it by the same symbol. Hence by Theorem 3.20, $\gamma$ restricted to $\overline{\mathfrak{g}}$ is given as $\gamma_{\alpha, S}$ with $\alpha$ a suitable symmetric invariant bilinear form. Also by restriction to $\mathcal{L}$ we obtain $\gamma_{\mid \mathcal{L}}=r_{3} \gamma_{S, R}^{(v)}+\psi$ with $\psi$ a coboundary of $\mathcal{L}$. By the $\mathcal{L}$-invariance of $\gamma_{\alpha, S}$ all these cocycles can be extended by zero to the whole of $\mathcal{D}_{\mathfrak{g}}^{1}$ and we can consider the cocycle

$$
\gamma^{\prime}=\gamma-\left(\gamma_{\alpha, S}+r_{3} \gamma_{S, R}^{(v)}+\psi\right)
$$

It will vanish on pairs of elements of the same type. From (4.5) follows that $\gamma^{\prime}(e,[x, y](g))=$ 0 . Hence by decomposing $x \in \mathfrak{g}$ as $x=x_{0}+x^{\prime}$ with $x_{0} \in \mathfrak{g}_{0}$ and $x^{\prime} \in \mathfrak{g}^{\prime}$ we get $\gamma^{\prime}(e, x(g))=\gamma^{\prime}\left(e, x_{0}(g)\right)$. It follows that $\gamma^{\prime}$ is a local cocycle of $\mathcal{D}_{\mathrm{g}_{0}}^{1}$ of pure mixing type which is extended by zero on $\mathfrak{g}^{\prime}$. In particular, using Theorem 4.8, up to coboundary it can be given as $\gamma_{\phi, S}$ with $\phi \in \mathfrak{g}^{*}$ satisfying $\phi_{\mid \mathfrak{g}^{\prime}} \equiv 0$. This shows the first claim. Clearly, we obtain a local cocycle by any such linear combination. Altogether (using the results of Theorem 4.6 and Theorem 4.8) we obtain the statement (b) about the dimension of the cohomology space. 
A further consequence is:

Proposition 4.12. (a) Let $\gamma$ be a local cocycle of $\mathcal{D}_{\mathfrak{g}}^{1}$. There exists a linear form $\phi$ on $\mathfrak{g}$ which vanishes on $\mathfrak{g}^{\prime}$ and a linear form $\psi$ on $\overline{\mathfrak{g}}$ such that

$$
\gamma(e, x(g))=\phi(x) \gamma^{(m)}(e, g)+\psi(x(e . g)), \quad \forall e \in \mathcal{L}, g \in \mathcal{A}, x \in \mathfrak{g},
$$

with $\gamma^{(m)}$ a mixing cocycle of $\mathcal{D}^{1}$.

(b) Assume in addition that the cocycle $\gamma$ is $\mathcal{L}$-invariant if restricted to $\overline{\mathfrak{g}}$ (or equivalently to $\left.\overline{\mathfrak{g}}^{\prime}\right)$ then the linear form $\psi$ on $\overline{\mathfrak{g}}$ can be chosen such that it vanishes on $\overline{\mathfrak{g}}^{\prime}$.

Proof. (a) We use Theorem 4.11. If we evaluate $\gamma$ for pairs $(e, x(g))$ we obtain from (4.17) the expression (4.19). Note that every coboundary will be given by a linear form $\psi$ on $\mathcal{D}_{\mathfrak{g}}^{1}$, evaluated at the Lie bracket of the two elements. But this reduces exactly to the expression above.

(b) Now assume $\gamma$ to be $\mathcal{L}$-invariant if restricted to $\overline{\mathfrak{g}}$. Let $\gamma^{\prime}$ be the corresponding cohomologous cocycle of type (4.17). The difference will be a coboundary which evaluated at the relevant pairs will be $\delta \psi$. In particular it will be an $\mathcal{L}$-invariant cocycle. Assume that $\psi$ does not vanish on some element of the type $x(e . g)$ with $x \in \mathfrak{g}^{\prime}$, then $x=[y, z]$, $y, z \in \mathfrak{g}^{\prime}$. Hence $\psi([y, z](e . g)) \neq 0$ in contradiction to the assumed $\mathcal{L}$-invariance, see (4.5). Hence, by setting $\tilde{\psi}(x(h))=0$ for all $x \in \mathfrak{g}^{\prime}, h \in \mathcal{A}$ we obtain $\psi(x(h))=\tilde{\psi}(x(h))$ and get the required form in (4.19).

\section{EXAMPLES: $\mathfrak{s l}(n)$ AND $\mathfrak{g l}(n)$}

As examples we deal with the important special cases $\mathfrak{s l}(n)$ (which is a simple algebra) and the case of $\mathfrak{g l}(n)$ (which is reductive but not semi-simple). We will study the affine algebras, the differential operator algebras, and their central extensions. The corresponding algebras appear in the study of fermionic representations, see [44].

5.1. $\mathfrak{s l}(n)$. First we consider $\mathfrak{s l}(n)$, the Lie algebra of trace-less complex $n \times n$ matrices. Up to multiplication with a scalar the Cartan-Killing form $\beta(x, y)=\operatorname{tr}(x y)$ is the unique symmetric invariant bilinear form. It is non-degenerate.

Due to the fact that $\mathfrak{s l}(n)$ is simple, from Theorem 3.13 and Theorem 4.6 follows

Proposition 5.1. (a) Every local cocycle for the current algebra $\overline{\mathfrak{s l}}(n)$ is cohomologous to

$$
\gamma(x(g), y(h))=r \frac{\operatorname{tr}(x y)}{2 \pi \mathrm{i}} \int_{C_{S}} g d h, \quad r \in \mathbb{C} .
$$

(b) Every $\mathcal{L}$-invariant local cocycle equals the cocycle (5.1) with a suitable $r$.

(c) Every local cocycle for the differential operator algebra $\mathcal{D}_{\mathfrak{s r}(n)}^{1}$ is cohomologous to a linear combination of (5.1) and the standard local cocycle $\gamma_{S, R}^{(v)}$ for the vector field algebra. In particular, there exist no cocycles of pure mixing type. 
5.2. $\mathfrak{g l}(n)$. Next we deal with $\mathfrak{g l}(n)$, the Lie algebra of all complex $n \times n$-matrices. Recall that $\mathfrak{g l}(n)$ can be written as Lie algebra direct sum $\mathfrak{g l}(n)=\mathfrak{s}(n) \oplus \mathfrak{s l}(n) \cong \mathbb{C} \oplus \mathfrak{s l}(n)$. Here $\mathfrak{s}(n)$ denotes the $n \times n$ scalar matrices. This decomposition is the decomposition as reductive Lie algebra into its abelian and semi-simple summand.

After tensoring with $\mathcal{A}$ we obtain $\overline{\mathfrak{g l}}(n)=\overline{\mathfrak{s}}(n) \oplus \overline{\mathfrak{s l}}(n) \oplus \cong \mathcal{A} \oplus \overline{\mathfrak{s l}}(n)$. From Theorem 3.20 it follows that we need to determine all symmetric invariant bilinear forms for $\overline{\mathfrak{g l}}(n)$. From the above decomposition it follows that the space of such forms is two-dimensional. An adapted basis is

$$
\alpha_{1}(x, y)=\operatorname{tr}(x y), \quad \text { and } \quad \alpha_{2}(x, y)=\operatorname{tr}(x) \operatorname{tr}(y) .
$$

The form $\alpha_{1}$ is the "natural" extension for the Cartan-Killing form for $\mathfrak{s l}(n)$ to $\mathfrak{g l}(n)$ and is also $\mathfrak{g l}(n)$ invariant. The form $\alpha_{2}$ vanishes on $\mathfrak{s l}(n)$ and is non-zero on $\mathfrak{s}(n)$. Note that $\alpha_{1 \mid \mathfrak{s}(n)} \not \equiv 0$. The by zero on $\mathfrak{s}(n)$ to $\mathfrak{g l}(n)$ extended form $\tilde{\alpha}_{1}$ of the Cartan-Killing form for $\mathfrak{s l}(n)$ would be

$$
\tilde{\alpha}_{1}(x, y)=\operatorname{tr}(x y)-\frac{1}{n} \operatorname{tr}(x) \operatorname{tr}(y) .
$$

From Theorem 3.20 we conclude

Proposition 5.2. (a) A cocycle for $\overline{\mathfrak{g l}}(n)$ is local and restricted to $\overline{\mathfrak{s}}$ is $\mathcal{L}$-invariant if and only if it is cohomologous to a linear combination of the following two cocycles

$$
\gamma_{1}(x(g), y(h))=\frac{\operatorname{tr}(x y)}{2 \pi \mathrm{i}} \int_{C_{S}} g d h, \quad \gamma_{2}(x(g), y(h))=\frac{\operatorname{tr}(x) \operatorname{tr}(y)}{2 \pi \mathrm{i}} \int_{C_{S}} g d h .
$$

(b) If the cocycle $\gamma$ of (a) restricted to $\overline{\mathfrak{g l}}(n)$ is $\mathcal{L}$-invariant then $\gamma$ is equal to the linear combination of the cocycles (5.4)

Proposition 5.3. (a) Every local cocycle $\gamma$ for $\mathcal{D}_{\mathfrak{g l}(n)}^{1}$ is cohomologous to a linear combination of the cocycles $\gamma_{1}$ and $\gamma_{2}$ of (5.4), of the mixing cocycle

$$
\gamma_{3, T}(e, x(g))=\frac{\operatorname{tr}(x)}{2 \pi \mathrm{i}} \int_{C_{S}}\left(\tilde{e} g^{\prime \prime}+T \tilde{e} g^{\prime}\right) d z
$$

and of the standard local cocycle $\gamma_{S, R}^{(v)}$ for the vector field algebra, i.e.

$$
\gamma=r_{1} \gamma_{1}+r_{2} \gamma_{2}+r_{3} \gamma_{3, T}+r_{4} \gamma_{S, R}^{(v)}+\text { coboundary }
$$

with suitable $r_{1}, r_{2}, r_{3}, r_{4} \in \mathbb{C}$.

(b) If the cocycle $\gamma$ is local and restricted to $\overline{\mathfrak{g l}}(n)$ is $\mathcal{L}$-invariant, and $r_{3}, r_{4} \neq 0$ then there exist an affine connection $T^{\prime}$ and a projective connection $R^{\prime}$ holomorphic outside A such that $\gamma=r_{1} \gamma_{1}+r_{2} \gamma_{2}+r_{3} \gamma_{3, T^{\prime}}+r_{4} \gamma_{S, R^{\prime}}^{(v)}$.

(c) $\operatorname{dim} \mathrm{H}_{l o c}^{2}\left(\mathcal{D}_{\mathfrak{g l}(n)}^{1}, \mathbb{C}\right)=4$.

Proof. For $\mathfrak{g l}(n)=\mathfrak{s}(n) \oplus \mathfrak{s l}(n)$ up to multiplication with a scalar, the unique linear form vanishing on $\mathfrak{s l}(n)$ is $x \mapsto \operatorname{tr}(x)$. Hence from Proposition 5.2 and Theorem 4.11 the claim (a) follows. To prove (b) we have to note that by the $\mathcal{L}$-invariance using 
Proposition 5.2(b) it follows that the restriction to $\overline{\mathfrak{g l}}(n)$ is without changing the element in the cohomology class already of the required type. Also by the non-vanishing of the coefficients $r_{3}$ and $r_{4}$ the coboundaries can be included into the definition of $\gamma_{S, R^{\prime}}^{(v)}$ and $\gamma_{3, T^{\prime}}$ by choosing suitable projective and affine projections. Part (c) follows from Theorem 4.11, Part (b).

For the cocycle obtained by the regularization of the fermionic representations associated to $\mathfrak{g l}(n)$ its mixing part was studied by Sheinman in 45. Independent of the results in [38, he obtains results on its structure.

\section{Appendix A. Proof of Lemma 3.18}

In this appendix I give the details of the proof of Lemma 3.18 by recalling the steps of the proof of the uniqueness for the local and $\mathcal{L}$-invariant cocycles for the function algebra $\mathcal{A}$ (see [38, Sections 5.1 and 5.2]) now suitable adjusted to our more general situation. Let $\gamma$ be an $\mathcal{L}$-invariant and local cocycle for the current algebra $\overline{\mathfrak{g}}=\mathfrak{g} \otimes \mathcal{A}$ of an abelian finite-dimensional Lie algebra $\mathfrak{g}$. We fix $x, y \in \mathfrak{g}$ and set $\gamma_{x, y}(f, g):=\gamma(x \otimes f, y \otimes g)$. This will define a bilinear form on $\mathcal{A}$. A priori we do not know that $\gamma_{x y}$ will be antisymmetric. By the $\mathcal{L}$-invariance we obtain

$$
\gamma_{x y}(e \cdot f, g)+\gamma_{x y}(f, e \cdot g)=0, \quad \forall e \in \mathcal{L}, \forall f, g \in \mathcal{A} .
$$

Our aim is to show that we can choose $\alpha_{x y} \in \mathbb{C}$ such that $\gamma_{x y}\left(A_{m, s}, A_{l, t}\right)=\alpha_{x y} \gamma_{S}^{(f)}\left(A_{m, s}, A_{l, t}\right)$ for all pairs of the basis elements $\left\{A_{m, s} \mid m \in \mathbb{Z}, s=1, \ldots, K\right\}$ of $\mathcal{A}$.

By the almost-graded action of $\mathcal{L}$ on $\mathcal{A}$ we have

$$
e_{n, r} \cdot A_{m, s}=\delta_{r}^{s} \cdot m \cdot A_{n+m, r}+\sum_{h=n+m+1}^{n+m+L_{2}} \sum_{t=1}^{K} b_{(n, r),(m, s)}^{(h, t)} A_{h, t},
$$

with $b_{(n, r),(m, s)}^{(h, t)} \in \mathbb{C}$, and $L_{2}$ the upper bound for the almost-graded structure. Equation (A.1) for basis elements writes as

$$
\gamma_{x y}\left(e_{n, r} \cdot A_{m, s}, A_{p, t}\right)+\gamma_{x y}\left(A_{m, s}, e_{n, r} \cdot A_{p, t}\right)=0 .
$$

Using (A.2) we get

$$
\delta_{r}^{s} \cdot m \cdot \gamma_{x y}\left(A_{m+n, r}, A_{p, t}\right)+\delta_{r}^{t} \cdot p \cdot \gamma_{x y}\left(A_{m, s}, A_{n+p, t}\right)=\text { h.l. }
$$

Here and in the following I will use the phrase "can be expressed by elements of higher level", "determined by higher level", or simply "= h.l." to denote that it is a universal linear combination of values for pairs of homogeneous elements of levels higher than the level under consideration. By the level I understand the sum of the degrees of the two arguments. The coefficients appearing in this linear combination only depend on the geometric situation, i.e. on the structure constants of the algebra and not on the the bilinear form under consideration. In particular, if two bilinear forms are given by higher level and they coincide in higher levels, they will coincide also for the elements under consideration. 
For $r=t \neq s$ we obtain

$$
p \cdot \gamma_{x y}\left(A_{m, s}, A_{n+p, t}\right)=\text { h.l. }
$$

Hence, as long as $s \neq t$ the value $\gamma_{x y}\left(A_{m, s}, A_{l, t}\right)$ is determined by higher level. Next we consider $s=t=r$. From (A.4) it follows

$$
m \cdot \gamma_{x y}\left(A_{m+n, r}, A_{p, r}\right)+p \cdot \gamma_{x y}\left(A_{m, r}, A_{n+p, r}\right)=\text { h.l. }
$$

We set $n=0$ and obtain

$$
(m+p) \cdot \gamma_{x y}\left(A_{m, r}, A_{p, r}\right)=\text { h.l. }
$$

Hence, for $p \neq-m$ the value $\gamma_{x y}\left(A_{m, r}, A_{p, r}\right)$ is determined by higher level.

Next in A.6) we set $p=-(n+1), m=1$ and obtain

$$
\gamma_{x y}\left(A_{n+1, r}, A_{-(n+1), r}\right)=(n+1) \gamma_{x y}\left(A_{1, r}, A_{-1, r}\right)+\text { h.l. }
$$

Now we use that $\gamma$ is local. In particular, it is bounded from above. Starting from a certain level the value of the bilinear form will be zero if evaluated for elements of level higher than this level. But by the above inductive procedure it has to vanish for all levels greater zero.

Let $C_{i}$ be a (possibly deformed) circle around the incoming point $P_{i} \in I$. We set $\gamma_{i}^{(f)}(f, g):=\frac{1}{2 \pi \mathrm{i}} \int_{C_{i}} f d g$. This is a cocycle for $\mathcal{A}$ which is bounded from above by zero. Obviously, for the separating cocycle $\gamma_{S}^{(f)}=\sum_{i=1}^{K} \gamma_{i}^{(f)}$. Note that $\gamma_{i}^{(f)}$ is not necessarily bounded from below and hence not necessarily local. By calculations of residues we obtain

$$
\gamma_{i}^{(f)}\left(A_{n, r}, A_{-n, r}\right)=-n \cdot \delta_{r}^{i} .
$$

Set $\tilde{\gamma}=\sum_{r=1}^{K} \alpha_{x y}^{r} \gamma_{r}^{(f)}$ with $\alpha_{x y}^{r}:=-\gamma_{x y}\left(A_{1, r}, A_{-1, r}\right) \in \mathbb{C}$. At level zero (and higher) $\tilde{\gamma}=\gamma_{x y}$, and hence by induction at every level. In particular $\gamma_{x y}$ is a local cocycle and we can apply the results of 38. By Theorem 4.3 in 38, $\gamma_{x y}$ will be local if and only if in above linear combination all coefficients are the same, i.e. $\alpha_{x y}:=\alpha_{x y}^{1}=\alpha_{x y}^{2}=\cdots=\alpha_{x y}^{K}$. Hence, $\gamma_{x y}=\alpha_{x y} \gamma_{S}^{(f)}$. This proves Lemma 3.18.

\section{REFERENCES}

[1] E. Arbarello, C. De Concini, V.G. Kac, and C. Procesi, Moduli spaces of curves and representation theory, Commun. Math. Phys. 117 (1988), no. 1, 1-36.

[2] K. Bardakçi and M.B. Halpern, New dual quark models., Phys. Rev. D 3 (1971), 2493.

[3] S. Berman, and Y. Billig, Irreducible representations for toroidal Lie algebras J. Algebra 221 (1999), 188-231.

[4] S. Berman and I. Kryliouk, Universal central extensions of some Lie algebras., Futorny, V. (ed.) et al., Modern trends in Lie algebra representation theory. Queen's Pap. Pure Appl. Math. 94, 19-23, Queen's Univ. Press., 1994.

[5] N. Bourbaki, Elements of mathematics. Lie groups and Lie algebras, Springer Verlag, Berlin, 1989.

[6] M. Bremner, Universal central extensions of elliptic affine Lie algebras, J. Math. Phys. 35 (1994), no. $12,6685-6692$.

[7] M. Bremner, Four-point affine Lie algebras, Proc. Am. Math. Soc. 123 (1995), no. 7, 1981-1989. 
[8] P. I. Etingof and I. B. Frenkel, Central extensions of current groups in two dimensions., Commun. Math. Phys. 165 (1994), no. 3, 429-444.

[9] B. L. Feigin, Cohomology of groups and current algebras., Usp. Mat. Nauk, 35 (1980), 224-226.

[10] B. L. Feigin, Conformal field theory and cohomologies of the Lie algebra of holomorphic vector fields on a complex curve., Proc. ICM, Kyoto, Japan (1990), 71-85.

[11] I. B. Frenkel and B. Khesin, Four dimensional realization of two-dimensional current groups. Commun. Math. Phys. 178 (1996), 541-561.

[12] J. Fuchs and Ch. Schweigert, Symmetries, Lie algebras and representations. A graduate course for physicists., Cambridge Monographs on Mathematical Physics. Cambridge University Press, 1997.

[13] D.B. Fuks, Cohomology of infinite-dimensional Lie algebras. Transl. from the Russian by A. B. Sosinskij., Contemporary Soviet Mathematics. New York: Consultants Bureau. XII, 1986.

[14] H. Garland, The arithmetic theory of loop groups., Publ. Math., Inst. Hautes Etud. Sci. 52 (1980), $5-136$.

[15] Ph. Griffiths and J. Harris, Principles of algebraic geometry, John Wiley, New York, 1978.

[16] A. Haddi, Homologie des algèbres de Lie étendues à une algèbre commutative., Commun. Algebra 20 (1992), no. 4, 1145-1166.

[17] V. G. Kac, Simple irreducible graded Lie algebras of finite growth. (Russian)., Izv. Akad. Nauk SSSR Ser. Mat. 32 (1968), 1323-1367.

[18] V. G. Kac, Infinite dimensional Lie algebras. 3rd ed., Cambridge University Press, 1990.

[19] Ch. Kassel, Kähler differentials and coverings of complex simple Lie algebras extended over a commutative algebra, J. Pure Appl. Algebra 34 (1984), 265-275.

[20] Ch. Kassel and J-L. Loday, Extensions centrales d'algébres de Lie., Ann. Inst. Fourier 32 (1982), no. 4, 119-142.

[21] N. Kawazumi, On the complex analytic Gel'fand-Fuks cohomology of open Riemann surfaces, Ann. Inst. Fourier 43 (1993), no. 3, 655-712.

[22] I.M. Krichever and S.P. Novikov, Algebras of Virasoro type, Riemann surfaces and structures of the theory of solitons, Funktional Anal. i. Prilozhen. 21 (1987), 46-63.

[23] I.M. Krichever and S.P. Novikov, Virasoro type algebras, Riemann surfaces and strings in Minkowski space, Funktional Anal. i. Prilozhen. 21 (1987), 47-61.

[24] I.M. Krichever and S.P. Novikov, Algebras of Virasoro type, energy-momentum tensors and decompositions of operators on Riemann surfaces, Funktional Anal. i. Prilozhen. 23 (1989), 46-63.

[25] T.A. Larsson, Extensions of diffeomorphism and current algebras, math-ph/0002016 (2000).

[26] D.V. Millionshchikov, Krichever-Novikov algebras and the cohomology of the algebra of meromorphic vector fields, Amer. Math. Soc. Transl. 179 (1997), 101-108.

[27] R.V. Moody, S.E. Rao, and T. Yokonoma, Toroidal Lie algebras and vertex representations. Geom. Ded. 35 (1990), 283-307.

[28] A. Pressley and G. Segal, Loop Groups, Clarendon Press, Oxford, 1986.

[29] C. Roger, Extensions centrales d'algèbres et de groupes de Lie de dimension infinie, algèbre de Virasoro et généralisations. Rep. Math. Phys. 35 (1995), 225-266.

[30] V.A. Sadov, Bases on multipunctured Riemann surfaces and interacting strings amplitudes, Commun. Math. Phys. 136 (1991), 585-597.

[31] L.J. Santharoubane, The second cohomology group for Kac-Moody Lie algebras and Kähler differentials., J. Algebra 125 (1989), no. 1, 13-26.

[32] M. Schlichenmaier, Introduction to Riemann surfaces, algebraic curves and moduli spaces, Lecture Notes in Physics, vol. 322, Springer, Berlin, Heidelberg, New York, 1990.

[33] M. Schlichenmaier, Verallgemeinerte Krichever - Novikov Algebren und deren Darstellungen, Ph.D. thesis, Universität Mannheim, 1990. 
[34] M. Schlichenmaier, Central extensions and semi-infinite wedge representations of KricheverNovikov algebras for more than two points, Lett. Math. Phys. 20 (1991), 33-46.

[35] M. Schlichenmaier, Differential operator algebras on compact Riemann surfaces, Generalized Symmetries in Physics (Clausthal 1993, Germany) (H.-D. Doebner, V.K. Dobrev, and A.G. Ushveridze, eds.), World Scientific, 1994.

[36] M. Schlichenmaier, Zwei Anwendungen algebraisch-geometrischer Methoden in der theoretischen Physik: Berezin-Toeplitz-Quantisierung und globale Algebren der zweidimensionalen konformen Feldtheorie, Habilitation thesis, 1996.

[37] M. Schlichenmaier, Sugawara operators for higher genus Riemann surfaces, Rep. on Math. Phys. 43 (1999), 323-339, math.QA/9806032

[38] M. Schlichenmaier, Local cocycles and central extensions for multi-point algebras of KricheverNovikov type, math/0112116 (to appear in Jour. Reine u. Angewandte Math.).

[39] M. Schlichenmaier, Algebras of meromorphic differential operators on higher genus Riemann surfaces and semi-infinite wedge representations, in preparation.

[40] M. Schlichenmaier and O.K. Sheinman, The Sugawara construction and Casimir operators for Krichever-Novikov algebras., J. Math. Sci., New York 92 (1998), no. 2, 3807-3834.

[41] M. Schlichenmaier and O.K. Sheinman, Wess-Zumino-Witten-Novikov theory, KnizhnikZamolodchikov equations, and Krichever-Novikov algebras, I., Russian Math. Surv. (Uspeki Math. Naukii) 54 (1999), 213-250, math.QA/9812083

[42] O.K. Sheinman, Elliptic affine Lie algebras, Funct. Anal. Appl. 24 (1990), no. 3, 210-219.

[43] O.K. Sheinman, Affine Lie algebras on Riemann surfaces, Funct. Anal. Appl. 27 (1993), no. 4, $266-272$.

[44] O.K. Sheinman, A fermion model of representations of affine Krichever-Novikov algebras, Funct. Anal. Appl. 35 (2001), no. 3, 209-219.

[45] O.K. Sheinman, Second order Casimirs for the affine Krichever-Novikov algebras $\widehat{g l}_{g, 2}$ and $\widehat{s l}_{g, 2}$, Moscow Mathematical Journal 1 (2001), no. 4, 605-628.

[46] F. Wagemann, Some remarks on the cohomology of Krichever-Novikov algebras, Lett. Math. Phys. 47 (1999), no. 2, 173-177; Erratum: Lett. Math. Phys. 52(2000), 349.

[47] F. Wagemann, Density of meromorphic in holomorphic vector fields, preprint 2001.

[48] P. Zusmanovich, The second homology group of current Lie algebras., Kassel, Christian (ed.) et al., K-theory., S.M.F., Astérisque. 226, 435-452, 1994.

(Martin Schlichenmaier) Department of Mathematics and Computer Science, University of Mannheim, A5, D-68131 Mannheim, Germany

E-mail address: schlichenmaier@math.uni-mannheim.de 